\title{
COHOMOLOGY OF PRODUCTS AND COPRODUCTS OF AUGMENTED ALGEBRAS
}

\author{
MATTHEW TOWERS
}

\begin{abstract}
We show that the ordinary cohomology functor $\Lambda \mapsto \operatorname{Ext}_{\Lambda}^{*}(k, k)$ from the category of augmented $k$-algebras to itself exchanges coproducts and products, then that Hochschild cohomology is close to sending coproducts to products if the factors are self-injective. We identify the multiplicative structure of the Hochschild cohomology of a product, modulo a certain ideal, in terms of the cohomology of the factors.
\end{abstract}

\section{INTRODUCTION}

An augmented $k$-algebra is a $k$-algebra $\Lambda$ equipped with a homomorphism $\Lambda \rightarrow k$. The aim of this paper is to study how the ordinary cohomology $\operatorname{Ext}_{\Lambda}^{*}(k, k)$ and the Hochschild cohomology $\mathrm{HH}(\Lambda)$ of such algebras interact with products and coproducts.

The product in the category of augmented algebras is a pullback, like the fiber product of local rings from commutative algebra [Moo09]. Products can also occur naturally in a non-commutative setting, for example as endomorphism rings of certain permutation modules for group algebras (Example 2.4). The coproduct construction is similar to the free product of groups, for which some cohomological results are known [HS71, VI.14] which bear a similarity to our results on Hochschild cohomology of coproducts.

Results on the cohomology of coproducts and products for certain classes of algebras have already appeared in the literature, for example for graded algebras $A$ with $A_{0}=k$ in [PP05, Proposition 1.1], for local Noetherian commutative algebras over a field in [Moo09], and for coproducts of groups in [HS71, VI.14].

This paper begins with a brief overview of the category $\mathcal{A}$ of augmented $k$ algebras in Section 2, including its product and coproduct. We collect some useful information on Hochschild cohomology in Section 3. In Sections 4 and 5 we look at cohomology of coproducts, showing in Theorem 4.1 that the contravariant functor $E$ from $\mathcal{A}$ to itself sending $\Lambda$ to $\operatorname{Ext}_{\Lambda}^{*}(k, k)$ maps coproducts to products, and in Theorem 5.2 that Hochschild cohomology very nearly does the same thing if the factors are self-injective. These results are obtained easily using dimension shifting. Finally in Section 6 we look at the cohomology of products of augmented algebras. Given two augmented algebras $\Lambda$ and $\Gamma$ we construct a 'small' bimodule resolution of the product $\Lambda * \Gamma$ from small bimodule resolutions of the factors. Here a resolution $P$ being small means that the image of the differential is contained in $I \cdot P$ where $I$ is the augmentation ideal - if $\Lambda$ is a finite-dimensional local algebra then this is equivalent to the resolution being minimal in the usual sense. Our resolution can be used to study ordinary cohomology, and we use it in Theorem 6.9 to prove that the functor $E$ given above sends products to coproducts. We then use a long exact sequence to investigate the Hochschild cohomology of $\Lambda * \Gamma$. This is a long way from being the coproduct of the Hochschild cohomology algebras of its factors: there is no hope of this since Hochschild cohomology is graded commutative. In Theorem 6.15 we show that, modulo a certain ideal, $\mathrm{HH}(\Lambda * \Gamma)$ is the direct sum of 
the product of certain subalgebras of $\mathrm{HH}(\Lambda)$ and $\mathrm{HH}(\Gamma)$ with terms related to $E(\Lambda)$ and $E(\Gamma)$. We also show in Proposition 6.14 that if $I(\Lambda)$ and $I(\Gamma)$ are nilpotent then every element of positive degree in the Hochschild cohomology of the product $\Lambda * \Gamma$ is nilpotent with nilpotency degree at most the maximum of the nilpotency degrees of the augmentation ideals of the two factors.

\section{The CATEgORY OF AUGMENTED ALGEBRAS}

Let $k$ be a field. We define the category $\mathcal{A}$ of augmented $k$-algebras as follows: an object of $\mathcal{A}$ is a pair $\left(\Lambda, \varepsilon_{\Lambda}\right)$ where $\Lambda$ is a unital $k$-algebra and $\varepsilon_{\Lambda}$ is an algebra homomorphism $\Lambda \rightarrow k$. We write $I(\Lambda)$ for $\operatorname{ker} \varepsilon_{\Lambda}$. A morphism in $\mathcal{A}$ between $\left(\Lambda, \varepsilon_{\Lambda}\right)$ and $\left(\Gamma, \varepsilon_{\Gamma}\right)$ is a $k$-algebra homomorphism $f: \Lambda \rightarrow \Gamma$ that preserves the augmentations, that is $\varepsilon_{\Lambda}=\varepsilon_{\Gamma} \circ f$ (so that $\mathcal{A}$ is a comma category in the sense of [Mac71, II.6]). An augmented algebra is an algebra that appears as the first coordinate of an object of $\mathcal{A}$.

Example 2.1. Any group algebra $k G$ is augmented with augmentation given by $g \mapsto 1$ for all $g \in G$. If $Q$ is a quiver and $I$ is an admissible ideal of $k Q$ then for each vertex e of $Q$ there is an augmentation $\varepsilon_{e}$ of $k Q / I$ sending (the image of) each arrow to zero, $e$ to 1 , and every other vertex to zero.

2.1. Products. In a comma category, the product can be defined using the pullback in the parent category. Given any two augmented algebras $\Lambda$ and $\Gamma$ we have a diagram

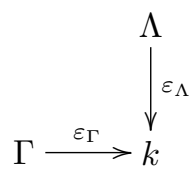

in the category of $k$-algebras.

Definition 2.2. $\Lambda * \Gamma$ is defined to be the pullback of (1) in the category of $k$ algebras.

Explicitly, $\Lambda * \Gamma$ is $\left\{(\lambda, \gamma) \in \Lambda \oplus \Gamma: \epsilon_{\Lambda} \lambda=\epsilon_{\Gamma} \gamma\right\}$, with the multiplication inherited from the direct sum $\Lambda \oplus \Gamma$. The pullback has projection maps $p_{\Lambda}: \Lambda * \Gamma \rightarrow \Lambda$ and $p_{\Gamma}: \Lambda * \Gamma \rightarrow \Gamma$. We can define an augmentation on $\Lambda * \Gamma$ by $\epsilon_{\Lambda * \Gamma}=\epsilon_{\Lambda} \circ p_{\Lambda}=\epsilon_{\Gamma} \circ p_{\Gamma}$.

Lemma 2.3. $\left(\Lambda * \Gamma, \epsilon_{\Lambda * \Gamma}\right)$ with canonical projections $p_{\Lambda}$ and $p_{\Gamma}$ is a product in $\mathcal{A}$ of $\left(\Lambda, \epsilon_{\Lambda}\right)$ and $\left(\Gamma, \epsilon_{\Gamma}\right)$.

For example, the product of $k[x] / x^{2}$ with itself is isomorphic to $k[x, y] /\left(x^{2}, y^{2}, x y\right)$.

Example 2.4. Let $k$ have characteristic 3 and let

$$
D=\left\langle x, y, z \mid x^{3}, y^{3}, z^{3},[x, y]=z \in Z(D)\right\rangle
$$

be the extra-special group of order 27 and exponent 3 . Then the endomorphism algebra of the transitive permutation module $k_{\langle x\rangle} \uparrow^{D}$ is

$$
k[a] / a^{3} * k[b] / b^{2} * k[c] / c^{2} .
$$

For further examples of this type see [Tow09].

This product satisfies the following Chinese Remainder-type result:

Lemma 2.5. Let $\Lambda$ be an augmented $k$-algebra with ideals $I, J$ such that $I+J=$ $I(\Lambda)$ and $I \cap J=I J$. Then $\Lambda / I J \cong \Lambda / I * \Lambda / J$. 
2.2. Coproducts. The coproduct in $\mathcal{A}$ is a free product construction like that in the category of groups. Given augmented $k$-algebras $\Lambda$ and $\Gamma$ let $R_{0}=k$ and $R_{n}=\left(I(\Lambda) \otimes_{k} I(\Gamma) \otimes_{k} \ldots\right) \oplus\left(I(\Gamma) \otimes_{k} I(\Lambda) \otimes_{k} \ldots\right)$ where both summands have length $n>0$.

Definition 2.6. $\Lambda \sqcup \Gamma$ is defined to be $\bigoplus_{n \geq 0} R_{n}$ equipped with the multiplication

$$
\left(u_{1} \otimes \cdots \otimes u_{k}\right) \cdot\left(v_{1} \otimes \cdots \otimes v_{l}\right)=u_{1} \otimes \cdots \otimes\left(u_{k} v_{1}\right) \otimes v_{2} \otimes \cdots \otimes v_{l}
$$

if $u_{k}$ and $v_{1}$ are both in $\Lambda$ or both in $\Gamma$, and

$$
u_{1} \otimes \cdots \otimes u_{k} \otimes v_{1} \otimes v_{2} \otimes \cdots \otimes v_{l}
$$

otherwise.

$\Lambda \sqcup \Gamma$ has an augmentation $\epsilon_{\Lambda \sqcup \Gamma}$ which quotients out all $R_{n}$ with $n>0$. There are injective algebra homomorphisms $i_{\Lambda}: \Lambda \rightarrow \Lambda \sqcup \Gamma$ and $i_{\Gamma}: \Gamma \rightarrow \Lambda \sqcup \Gamma$ which send elements of $\Lambda$ and $\Gamma$ to their images in $R_{0} \oplus R_{1} \subset \Lambda \sqcup \Gamma$.

Lemma 2.7. ( $\left.\Lambda \sqcup \Gamma, \epsilon_{\Lambda \sqcup \Gamma)}\right)$ with canonical injections $i_{\Lambda}$ and $i_{\Gamma}$ is a coproduct in $\mathcal{A}$ of $\left(\Lambda, \epsilon_{\Lambda}\right)$ and $\left(\Gamma, \epsilon_{\Gamma}\right)$

For example, $k[x] \sqcup k[y]$ is the free associative algebra on two variables.

Remark 2.8. A $k$-algebra $\Lambda$ is said to be local if it has a unique maximal left ideal $\mathfrak{m}$ such that $\Lambda / \mathfrak{m} \cong k$ (any such ideal is automatically two-sided). Let $\mathcal{L}$ be the category of local algebras, where the morphisms are homomorphisms of algebras. Then the obvious inclusion is a fully faithful embedding $\mathcal{L} \hookrightarrow \mathcal{A}$ (fullness follows because any map of local algebras automatically preserves the maximal ideals). * induces a product on $\mathcal{L}$, but $\Lambda \sqcup \Gamma$ may fail to be local even if $\Lambda$ and $\Gamma$ are local: for example the coproduct in $\mathcal{A}$ of the local algebras $k[x] / x^{2}$ and $k[y] / y^{2}$ is $k\langle x, y\rangle /\left(x^{2}, y^{2}\right)$, which is not local as neither $x y$ nor $1-x y$ has a left-inverse. Equipping $*$ with injections $j_{\Lambda}: \Lambda \rightarrow \Lambda * \Gamma$ defined by $j_{\Lambda}(1)=(1,1)$ and $j_{\Lambda}(\lambda)=(\lambda, 0)$ for $\lambda \in I_{\Lambda}$ and $j_{\Gamma}$ defined similarly does not make it induce a coproduct on $\mathcal{L}$ : in the diagram

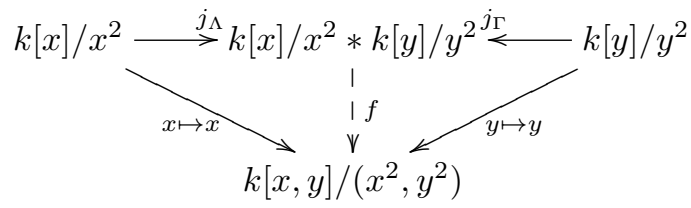

any $f$ making the triangles commute must send $j_{\Lambda}(x)$ to $x$ and $j_{\Gamma}(y)$ to $y$, but such an $f$ is not a map of algebras as $f\left(j_{\Lambda}(x) \cdot j_{\Gamma}(y)\right)=f(0)$ while $f\left(j_{\Lambda}(x)\right) \cdot f\left(j_{\Gamma}(y)\right)=$ $x y \neq 0$. We conjecture that in general $\mathcal{L}$ does not have a coproduct of $k[x] / x^{2}$ and $k[y] / y^{2}$.

The one-dimensional algebra $k$ is both an initial object (empty product) and terminal object (empty coproduct) in $\mathcal{A}$.

2.3. The ordinary cohomology functor $E$. Write $E(\Lambda)$ for the ordinary cohomology ring $\operatorname{Ext}_{\Lambda}^{*}(k, k)$, augmented by the map killing all elements of positive degree, and write $E^{n}(\Lambda)$ for $\operatorname{Ext}_{\Lambda}^{n}(k, k)$. Change of rings [CE56, VIII, §3] can be used to make $E$ into a contravariant functor on $\mathcal{A}$. Let $f$ be an augmentation preserving map $\Lambda \rightarrow \Gamma$, so that $E(f)$ should be a map $E(\Gamma) \rightarrow E(\Lambda)$. The definition of $E(f)$ is particularly straightforward when we regard $\operatorname{Ext}_{\Gamma}^{n}(k, k)$ as the set of equivalence classes of exact sequences of $\Gamma$-modules of the form

$$
0 \rightarrow k \rightarrow N_{n-1} \rightarrow \cdots \rightarrow N_{0} \rightarrow k \rightarrow 0 .
$$

$E(f)$ simply maps the class of this sequence to the class of the same sequence regarded as a sequence of $\Lambda$-modules via $f$. More commonly we will define $E(f)$ as 
follows: take projective resolutions $P_{*}$ and $Q_{*}$ of $k$ as a $\Lambda$ - and $\Gamma$-module respectively. The $Q_{n}$ become $\Lambda$-modules via $f$, so there is a chain map $H_{*}$ lifting the identity $k \rightarrow k$ :

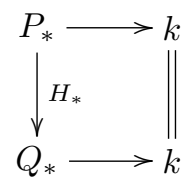

To define $E(f)$ on an element $a$ of $\operatorname{Ext}_{\Gamma}^{n}(k, k)$ we pick a representative cocycle $\alpha: Q_{n} \rightarrow k$ and let $E(f)(a)$ be the cohomology class of $\alpha \circ H_{n}: P_{n} \rightarrow k$.

Theorem 4.1 and Theorem 6.9 amount to saying that the functor $E$ preserves certain (co)limits. This would be immediate if a left (right) adjoint to $E$ existed.

Lemma 2.9. E has no right or left adjoint.

Proof. Recall that $E\left(k[x] / x^{2}\right)=k[a]$ with $a$ in degree one, and for $r>2, E\left(k[x] / x^{r}\right)=$ $k[a, b] / a^{2}$ with $a$ in degree one and $b$ in degree two.

If a contravariant functor has a left adjoint, it sends monomorphisms to epimorphisms. Apply $E$ to the monomorphism $f: k[x] / x^{2} \rightarrow k[x] / x^{4}$ that sends $x$ to $x^{2}$. The resulting map $E(f): k[a, b] / a^{2} \rightarrow k[a]$ is zero in degree one, and is not an epimorphism (although epimorphisms in $\mathcal{A}$ need not be onto, composing with the zero and quotient maps $k[a] \rightarrow k[a] / a^{2}$ gives the same result).

If $E$ had a right adjoint it would have to send epimorphisms to monomorphisms. This time let $f$ be the epimorphism $k[x] / x^{3} \rightarrow k[x] / x^{2}$ given by $x \mapsto x$. Then $E(f)$ is zero in degree one, therefore not a monomorphism (the monomorphisms in $\mathcal{A}$ are exactly the one-to-one homomorphisms).

\section{Hochschild COHOMOLOGY}

In this section we review some standard definitions and results about Hochschild cohomology.

Let $\Lambda$ be a $k$-algebra, and $\Lambda^{e}$ be the enveloping algebra $\Lambda \otimes_{k} \Lambda^{\mathrm{op}}$. Then $\Lambda$ is a left $\Lambda^{e}$-module via $(\lambda \otimes \gamma) \cdot x=\lambda x \gamma$. The categories of left $\Lambda^{e}$-modules and $\Lambda$ - $\Lambda$ bimodules are isomorphic, and we will often work with bimodules for convenience.

The Hochschild cohomology ring $\mathrm{HH}(\Lambda)$ is defined to be $\bigoplus_{n \geq 0} \operatorname{Ext}_{\Lambda^{e}}^{n}(\Lambda, \Lambda)$ equipped with the Yoneda product. $\mathrm{HH}(\Lambda)$ is a graded commutative $k$-algebra under the Yoneda product, and $\operatorname{HH}^{0}(\Lambda)$ is the centre $Z(\Lambda)$. We write $\operatorname{HH}^{n}(\Lambda)$ for $\operatorname{Ext}_{\Lambda^{e}}^{n}(\Lambda, \Lambda)$.

Hochschild cohomology as we have defined it is not functorial in $\Lambda$. Functoriality can be recovered by working instead with $\operatorname{Ext}_{\Lambda^{e}}^{*}\left(\Lambda, \operatorname{Hom}_{k}(\Lambda, k)\right.$ ) (this is done for example in [Lod98, Ben98]), the disadvantage of doing this is that there is no obvious ring structure. The two definitions coincide for symmetric algebras.

If $\Lambda$ is augmented then there is an exact sequence of $\Lambda$ - $\Lambda$-bimodules

$$
0 \rightarrow I(\Lambda) \rightarrow \Lambda \stackrel{\epsilon}{\rightarrow} k \rightarrow 0 .
$$

Applying $\operatorname{Hom}_{\Lambda^{e}}(\Lambda,-)$ gives a long exact sequence

$$
\cdots \rightarrow \operatorname{Ext}_{\Lambda^{e}}^{n}(\Lambda, I(\Lambda)) \rightarrow \mathrm{HH}^{n}(\Lambda) \stackrel{\phi_{k}}{\rightarrow} E^{n}(\Lambda) \stackrel{\omega_{\Lambda}}{\rightarrow} \operatorname{Ext}_{\Lambda^{e}}^{n+1}(\Lambda, I(\Lambda)) \rightarrow \cdots
$$

where we have made the identification $\operatorname{Ext}_{\Lambda^{e}}^{n}(\Lambda, k) \cong E^{n}(\Lambda)$ using [CE56, X, Theorem 2.1]. Here $\phi_{k}$ is the map in the long exact sequence arising from $\epsilon$, composed with this identification, and $\omega_{\Lambda}$ is the connecting homomorphism.

If $\Delta$ is a graded ring then the graded centre of $\Delta$ is defined to be the span of all homogeneous elements $z$ of $\Delta$ such that $z g=(-1)^{\operatorname{dg}(z) \operatorname{dg}(g)} g z$ for all homogeneous $g \in \Delta$. The map $\phi_{k}$ is a homomorphism of graded rings, and [SS04, Theorem 1.1] 
shows its image lies in the graded centre of $E(\Lambda)$ (in their notation, take $M=N=\Lambda$ and $\Gamma=k$ ). If $I(\Lambda)^{n}=0$, then the kernel of $\phi_{k}$ is nilpotent with each element having nilpotency index at most $n$ by [SS04, Proposition 4.4] — the result stated there is for the radical rather than the augmentation ideal, but if $I(\Lambda)^{n}=0$ they coincide.

\section{Ordinary COHOMOLOGY OF COPRODUCtS}

Theorem 4.1. Let $\Lambda$ and $\Gamma$ be augmented $k$-algebras. Then there is an isomorphism of algebras

$$
\operatorname{Ext}_{\Lambda \sqcup \Gamma}^{*}(k, k) \cong \operatorname{Ext}_{\Lambda}^{*}(k, k) * \operatorname{Ext}_{\Gamma}^{*}(k, k) .
$$

Proof. Notice that there is an isomorphism of $\Lambda \sqcup \Gamma$-bimodules

$$
I(\Lambda \sqcup \Gamma) \cong I(\Lambda) \uparrow \Lambda \sqcup \Gamma \oplus I(\Gamma) \uparrow \Lambda \sqcup \Gamma
$$

and also that $\Lambda \sqcup \Gamma$ is free on restriction to $\Lambda$ and $\Gamma$, regarded as subalgebras via the canonical injections $i_{\Lambda}$ and $i_{\Gamma}$. We can now compute the vector space structure of $E(\Lambda \sqcup \Gamma)$ using dimension shifting and the Eckmann-Shapiro lemma: for $n>0$,

$$
\begin{aligned}
\operatorname{Ext}_{\Lambda \sqcup \Gamma}^{n}(k, k) & \cong \operatorname{Ext}_{\Lambda \sqcup \Gamma}^{n-1}(I(\Lambda \sqcup \Gamma), k) \\
& \cong \operatorname{Ext}_{\Lambda \sqcup \Gamma}^{n-1}(I(\Lambda) \uparrow \Lambda \sqcup \Gamma, k) \oplus \operatorname{Ext}_{\Lambda \sqcup \Gamma}^{n-1}(I(\Gamma) \uparrow \Lambda \sqcup \Gamma, k) \\
& \cong \operatorname{Ext}_{\Lambda}^{n-1}(I(\Lambda), k) \oplus \operatorname{Ext}_{\Gamma}^{n-1}(I(\Gamma), k) \\
& \cong \operatorname{Ext}_{\Lambda}^{n}(k, k) \oplus \operatorname{Ext}_{\Gamma}^{n}(k, k) .
\end{aligned}
$$

It follows that $\operatorname{Ext}_{\Lambda \sqcup \Gamma}^{n}(k, k)$ and $\operatorname{Ext}_{\Lambda}^{n}(k, k) * \operatorname{Ext}_{\Gamma}^{n}(k, k)$ agree as vector spaces for all $n$. To show they have the same algebra structure we will prove that the algebra homomorphism $E\left(i_{\Lambda}\right) * E\left(i_{\Gamma}\right): E(\Lambda \sqcup \Gamma) \rightarrow E(\Lambda) * E(\Gamma)$ provided by the universal property of the product is an isomorphism.

By (4) there exist projective resolutions $\left(P_{*}, d_{*}^{P}\right)$ and $\left(Q_{*}, d_{*}^{Q}\right)$ of $k$ over $\Lambda$ and $\Gamma$ respectively with $P_{0}=\Lambda$ and $Q_{0}=\Gamma$ such that

$$
\cdots \rightarrow P_{2} \uparrow^{\Lambda \sqcup \Gamma} \oplus Q_{2} \uparrow^{\Lambda \sqcup \Gamma} \rightarrow P_{1} \uparrow \Lambda \sqcup \Gamma-Q_{1} \uparrow \Lambda \sqcup \Gamma \rightarrow \Lambda \sqcup \Gamma \stackrel{\epsilon_{\Lambda Ц \Gamma}}{\rightarrow} k \rightarrow 0
$$

is a projective resolution $\left((P * Q)_{*}, \delta_{*}\right)$ of $k$ over $\Lambda \sqcup \Gamma$. The differential $\delta_{*}$ in this complex is $d_{*}^{P \uparrow \Lambda \sqcup \Gamma} \oplus d_{*}^{Q} \uparrow \Lambda \sqcup \Gamma$, except at degree one where this is regarded as a map to $I(\Lambda) \uparrow \Lambda \sqcup \Gamma \oplus I(\Gamma) \uparrow \Lambda \sqcup \Gamma$ and composed with the isomorphism of (4) and the inclusion of $I(\Lambda \sqcup \Gamma)$ into $\Lambda \sqcup \Gamma$.

The universal property of induction gives a map of chain complexes of $\Lambda$-modules $j_{\Lambda}:\left.P_{*} \rightarrow P_{*} \uparrow \Lambda \sqcup \Gamma\right|_{\Lambda}$. There is also an inclusion of complexes $h_{\Lambda}: P_{\geq 1} \uparrow \Lambda \sqcup \Gamma \rightarrow P * Q$.

The chain map needed to compute $E\left(i_{\Lambda}\right)$ as in $(2)$ is $i_{\Lambda}$ in degree zero and $h_{\Lambda} \circ j_{\Lambda}$ in positive degrees.

We now take a chain map $\alpha_{*}$ on $P * Q$ representing an element of $E^{n}(\Lambda \sqcup \Gamma)$ with $n>0$ and follow its cohomology class through the isomorphisms of (5). The first simply restricts $\alpha_{*}$ to a chain map on the resolution of $I(\Lambda \sqcup \Gamma)$ obtained by truncating $P * Q$. Abusing notation we continue to refer to this map as $\alpha_{*}$. The second sends this to the class of $\left(\alpha_{*} \circ h_{\Lambda}, \alpha_{*} \circ h_{\Gamma}\right)$. The third is the EckmannShapiro isomorphism: it takes us to the class of $\left(\alpha_{*} \circ h_{\Lambda} \circ j_{\Lambda}, \alpha_{*} \circ h_{\Gamma} \circ j_{\Gamma}\right)$. The final isomorphism, like the first, is just a shift of degrees.

It follows that the isomorphism $\operatorname{Ext}_{\Lambda \sqcup \Gamma}^{n}(k, k) \cong \operatorname{Ext}_{\Lambda}^{n}(k, k) \oplus \operatorname{Ext}_{\Gamma}^{n}(k, k)$ of $(5)$ is equal to $E\left(i_{\Lambda}\right) \oplus E\left(i_{\Gamma}\right)$. The universal property of the product $E(\Lambda) * E(\Gamma)$ means that $E\left(i_{\Lambda}\right) * E\left(i_{\Gamma}\right): E(\Lambda \sqcup \Gamma) \rightarrow E(\Lambda) * E(\Gamma)$ satisfies

$$
\left(p_{E(\Lambda)} \oplus p_{E(\Gamma)}\right) \circ E\left(i_{\Lambda}\right) * E\left(i_{\Gamma}\right)=E\left(i_{\Lambda}\right) \oplus E\left(i_{\Gamma}\right)
$$

Since $\left(p_{E(\Lambda)}, p_{E(\Gamma)}\right)$ is an isomorphism in positive degrees, so is $E\left(i_{\Lambda}\right) * E\left(i_{\Gamma}\right)$. 


\section{HoChSCHILD COHOMOLOGY OF COPRODUCTS}

To investigate Hochschild cohomology of a coproduct using similar methods to the ones above, we need an analogue of (4) for bimodules.

Given an augmented algebra $A$ write $\Omega_{A^{e}}$ for the kernel of the bimodule map $d_{0}: A^{e} \rightarrow A$ defined by $a_{1} \otimes a_{2} \mapsto a_{1} a_{2}$. If $A$ is an augmented algebra then $\Omega_{A^{e}}$ is generated as an $A$ - $A$ bimodule by elements of the form $a \otimes 1-1 \otimes a$ for $a \in A$, by [CE56, IX, Proposition 3.1]. In fact if $S$ is a generating set for $A$ as an algebra then the elements $s \otimes 1-1 \otimes s$ for $s \in S$ generate $\Omega_{A^{e}}$ as a bimodule.

Lemma 5.1. There is an isomorphism of $(\Lambda \sqcup \Gamma)^{e}$-modules

$$
\Omega_{(\Lambda \sqcup \Gamma)^{e}} \cong \Omega_{\Lambda^{e} \uparrow}(\Lambda \sqcup \Gamma)^{e} \oplus \Omega_{\Gamma^{e} \uparrow}(\Lambda \sqcup \Gamma)^{e} .
$$

Proof. Consider the following diagram of $\Lambda \sqcup \Gamma$-bimodules.

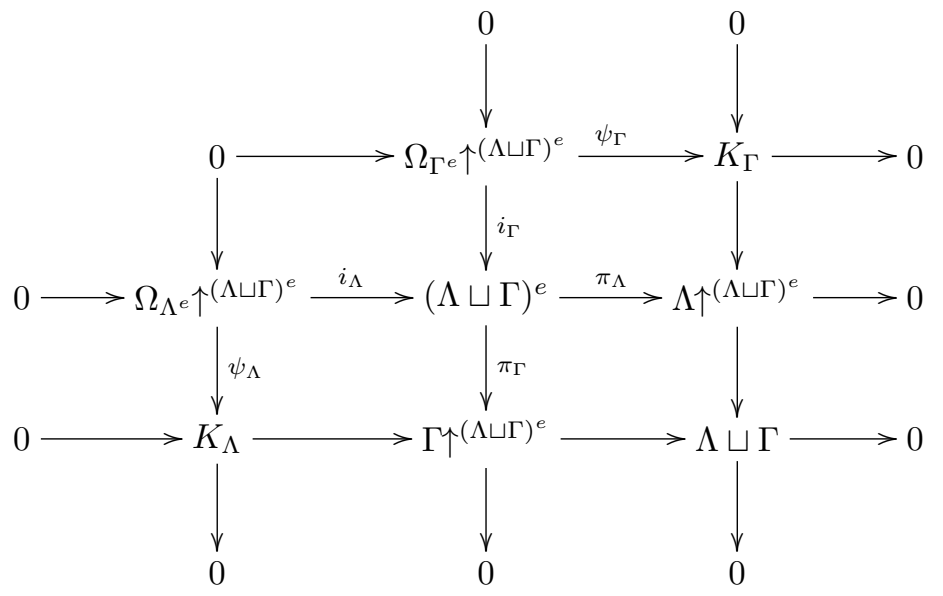

The middle row arises from applying the exact functor $\uparrow_{\Lambda^{e}}^{(\Lambda \sqcup \Gamma)^{e}}$ to

$$
0 \rightarrow \Omega_{\Lambda^{e}} \rightarrow \Lambda^{e} \rightarrow \Lambda \rightarrow 0
$$

and identifying $\Lambda^{e \uparrow(\Lambda \sqcup \Gamma)^{e}}$ with $(\Lambda \sqcup \Gamma)^{e}$ (the functor is exact because $(\Lambda \sqcup \Gamma)^{e}$ is free, hence flat, as a $\Lambda^{e}$-module). The middle column arises similarly. We identify $\Lambda \uparrow(\Lambda \sqcup \Gamma)^{e}$ with $(\Lambda \sqcup \Gamma) \otimes_{\Lambda}(\Lambda \sqcup \Gamma)$, so that the map $\pi_{\Lambda}:(\Lambda \sqcup \Gamma)^{e} \rightarrow \Lambda \uparrow(\Lambda \sqcup \Gamma)^{e}$ is $x \otimes y \mapsto x \otimes_{\Lambda} y$. The map $\Lambda \uparrow(\Lambda \sqcup \Gamma)^{e} \rightarrow \Lambda \sqcup \Gamma$ is $x \otimes_{\Lambda} y \mapsto x y$, and $K_{\Gamma}$ is defined to be its kernel. $K_{\Lambda}, \pi_{\Gamma}$, and the map $\Gamma \uparrow(\Lambda \sqcup \Gamma)^{e} \rightarrow \Lambda \sqcup \Gamma$ are defined similarly. $K_{\Lambda}$ is generated by the set $\left\{1 \otimes_{\Gamma} \lambda-\lambda \otimes_{\Gamma} 1: \lambda \in I(\Lambda)\right\}$.

Note that $K_{\Lambda}$ is free as a $\Gamma^{e}$-module. To see this, restrict the bottom row of the diagram to $\Gamma^{e}$ : we get

$$
\left.0 \rightarrow K_{\Lambda}\right|_{\Gamma^{e}} \rightarrow \Gamma \oplus F \rightarrow \Gamma \oplus G \rightarrow 0
$$

where $F$ and $G$ are free $\Gamma^{e}$-modules. The last non-zero map induces an isomorphism between the two $\Gamma$-summands, so if we delete these we get a short exact sequence in which the last two terms are free. Such a sequence must split, hence $\left.K_{\Lambda}\right|_{\Gamma^{e}}$ is free.

Let $M$ be a $\Lambda^{e}$-module. The universal property of the induced module $M \uparrow(\Lambda \sqcup \Gamma)^{e}$ is that there is a map of $\Lambda^{e}$-modules $\iota_{M}: M \rightarrow M \uparrow(\Lambda \sqcup \Gamma)^{e}$ such that given any $(\Lambda \sqcup \Gamma)^{e}$-module $N$ and a map $\alpha$ of $\Lambda^{e}$-modules $\left.M \rightarrow N\right|_{\Lambda^{e}}$ there is a unique map 
of $(\Lambda \sqcup \Gamma)^{e}$-modules $\hat{\alpha}: M \uparrow(\Lambda \sqcup \Gamma)^{e} \rightarrow N$ making the following diagram commute:

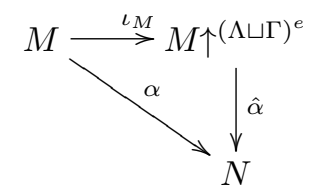

There is a map of $\Lambda^{e}$-modules $\beta: \Omega_{\Lambda^{e}} \rightarrow K_{\Lambda}$ which takes an element of $\Omega_{\Lambda^{e}} \subset \Lambda \otimes \Lambda$, regards it as an element of $(\Lambda \sqcup \Gamma)^{e}$ using the canonical injection, then applies the natural surjection $(\Lambda \sqcup \Gamma) \otimes_{k}(\Lambda \sqcup \Gamma) \rightarrow(\Lambda \sqcup \Gamma) \otimes_{\Gamma}(\Lambda \sqcup \Gamma)$. Then $\psi_{\Lambda}$ is the map $\hat{\beta}$ provided by the universal property of $\Omega_{\Lambda^{e} \uparrow}(\Lambda \sqcup \Gamma)^{e}$. Alternatively, $\psi_{\Lambda}$ is the map induced by the natural surjection $(\Lambda \sqcup \Gamma) \otimes_{k}(\Lambda \sqcup \Gamma) \rightarrow(\Lambda \sqcup \Gamma) \otimes_{\Gamma}(\Lambda \sqcup \Gamma)$.

We claim that the diagram is exact and commutative. Commutativity is easy to verify, and the only exactness that remains is showing that $\psi_{\Lambda}$ and $\psi_{\Gamma}$ are isomorphisms.

Note that $\beta$ is injective: the extra relations created by replacing $\otimes_{k}$ with $\otimes_{\Gamma}$ are spanned by tensors at least one of whose factors contains a term in $I(\Gamma)$. These cannot cause any extra linear dependences amongst tensors that only involve elements of $\Lambda$. Therefore $\beta\left(\Omega_{\Lambda^{e}}\right)$ is a $\Lambda^{e}$-submodule of $K_{\Lambda}$ isomorphic to $\Omega_{\Lambda^{e}}$. The $\Gamma^{e}$-submodule generated by $\beta\left(\Omega_{\Lambda^{e}}\right)$ is free on $\beta\left(\Omega_{\Lambda^{e}}\right)$ - if not, some element of $\beta\left(\Omega_{\Lambda^{e}}\right)$ would lie in $I\left(\Gamma^{e}\right) \cdot O_{\Lambda}$, which clearly does not happen.

This means that $K_{\Lambda}$ is freely generated as a $\Gamma^{e}$-module by the set of all elements of the form

$$
w_{1} \cdot \beta(n) \cdot w_{2}
$$

where $n$ is an element of some fixed basis of $\Omega_{\Lambda^{e}}$ and $w_{1}$ and $w_{2}$ are words in some fixed bases of $I(\Lambda)$ and $I(\Gamma)$ such that $w_{1}$ is empty or ends with an element of $I(\Gamma)$, and $w_{2}$ is empty or begins with an element of $I(\Gamma)$. Since $\Omega_{\Lambda^{e} \uparrow}(\Lambda \sqcup \Gamma)^{e}$ is free as a $\Gamma^{e}$-module on all such elements of the form $w_{1} \cdot n \cdot w_{2}$, it follows $\psi_{\Lambda}$ is an isomorphism.

$\operatorname{im} i_{\Lambda}+\operatorname{im} i_{\Gamma}$ is equal to $\Omega_{(\Lambda \sqcup \Gamma)^{e}}$ since it is a submodule containing all elements of the form $1 \otimes \lambda-\lambda \otimes 1$ for $\lambda \in I(\Lambda)$ and $1 \otimes \gamma-\gamma \otimes 1$ for $\gamma \in I(\Gamma)$. Also $\operatorname{im} i_{\Lambda} \cap \operatorname{im} i_{\Gamma}=\{0\}$, for if $i_{\Gamma}(y)$ lies in the intersection then it is killed by $\pi_{\Lambda}$, so by commutativity of the diagram $\psi_{\Gamma}(y)=0$ and therefore $y=0$. The result of the lemma follows.

5.1. First Hochschild cohomology of a coproduct. First Hochschild cohomology has to be dealt with separately, because the dimension shifting isomorphism in degree one is slightly different to that in higher degrees:

$$
\operatorname{Ext}_{(\Lambda \sqcup \Gamma)^{e}}^{1}(\Lambda \sqcup \Gamma, \Lambda \sqcup \Gamma) \cong \frac{\operatorname{Hom}_{(\Lambda \sqcup \Gamma)^{e}}\left(\Omega_{(\Lambda \sqcup \Gamma)^{e}}, \Lambda \sqcup \Gamma\right)}{F}
$$

where $F$ is the space of homomorphisms factoring through $\Omega_{(\Lambda \sqcup \Gamma)^{e}} \hookrightarrow(\Lambda \sqcup \Gamma)^{e}$.

Using Lemma 5.1 and the Eckmann-Shapiro lemma we have

$$
\begin{aligned}
\operatorname{HH}^{1}(\Lambda \sqcup \Gamma) & \cong \operatorname{Hom}_{(\Lambda \sqcup \Gamma)^{e}}\left(\Omega_{\Lambda^{e} \uparrow}(\Lambda \sqcup \Gamma)^{e} \oplus \Omega_{\Gamma^{e} \uparrow}(\Lambda \sqcup \Gamma)^{e}, \Lambda \sqcup \Gamma\right) / F \\
& \cong \frac{\operatorname{Hom}_{(\Lambda \sqcup \Gamma)^{e}}\left(\Omega_{\Lambda^{e} \uparrow}(\Lambda \sqcup \Gamma)^{e}, \Lambda \sqcup \Gamma\right) \oplus \operatorname{Hom}_{(\Lambda \sqcup \Gamma)^{e}}\left(\Omega_{\Gamma^{e} \uparrow}(\Lambda \sqcup \Gamma)^{e}, \Lambda \sqcup \Gamma\right)}{\left\{\left(f \circ \iota_{\Omega_{\Lambda} \uparrow^{(\Lambda \sqcup \Gamma)^{e}}}, f \circ \iota_{\left.\Omega_{\Gamma} \uparrow^{(\Lambda \sqcup \Gamma)^{e}}\right)}\right): f \in F\right\}} \\
& \cong \frac{\operatorname{Hom}_{\Lambda^{e}}\left(\Omega_{\Lambda^{e}}, \Lambda \oplus X\right) \oplus \operatorname{Hom}_{\Gamma^{e}}\left(\Omega_{\Gamma^{e}}, \Gamma \oplus Y\right)}{\left\{\left(f \circ \iota_{\Omega_{\Lambda^{e}}}, f \circ \iota_{\Omega_{\Gamma^{e}}}\right): f \in F\right\}}
\end{aligned}
$$

where $X$ and $Y$ are free $\Lambda^{e}$ - and $\Gamma^{e}$-modules respectively, $\iota_{\Omega_{\Lambda} \uparrow^{(\Lambda \sqcup \Gamma)^{e}}}$ is the inclusion of $\Omega_{\Lambda} \uparrow^{\uparrow}(\Lambda \sqcup \Gamma)^{e}$ into $\Omega_{(\Lambda \sqcup \Gamma)^{e}}$ that comes from Lemma 5.1 , and $\iota_{\Omega_{\Lambda} e}$ is the composition 
of this inclusion with the inclusion $\Omega_{\Lambda^{e}} \hookrightarrow \Omega_{\Lambda^{e}} \uparrow(\Lambda \sqcup \Gamma)^{e}$. Write $p_{\Lambda}$ and $p_{X}$ for the projections of $\Lambda \oplus X$ onto $\Lambda$ and $X$ respectively. Then $\operatorname{HH}^{1}(\Lambda \sqcup \Gamma)$ is isomorphic to

(6) $\frac{\operatorname{Hom}_{\Lambda^{e}}\left(\Omega_{\Lambda^{e}}, \Lambda\right) \oplus \operatorname{Hom}_{\Lambda^{e}}\left(\Omega_{\Lambda^{e}}, X\right) \oplus \operatorname{Hom}_{\Gamma^{e}}\left(\Omega_{\Gamma^{e}}, \Gamma\right) \oplus \operatorname{Hom}_{\Gamma^{e}}\left(\Omega_{\Gamma^{e}}, Y\right)}{\left\{\left(p_{\Lambda} \circ f \circ \iota_{\Omega_{\Lambda^{e}}}, p_{X} \circ f \circ \iota_{\Omega_{\Lambda^{e}}}, p_{\Gamma} \circ f \circ \iota_{\Omega_{\Gamma^{e}}}, p_{Y} \circ f \circ \iota_{\Omega_{\Gamma^{e}}}\right): f \in F\right\}}$

$F$ is spanned by maps $f_{w}: \Omega_{(\Lambda \sqcup \Gamma)^{e}} \rightarrow \Lambda \sqcup \Gamma$ for $w \in \Lambda \sqcup \Gamma$ given by

$$
f_{w}(x \otimes 1-1 \otimes x)=x w-w x
$$

for $x \in I(\Lambda \sqcup \Gamma)$. If $w \in I(\Lambda)$, the element of the denominator of (6) corresponding to $f_{w}$ is

$$
\left(p_{\Lambda} \circ f_{w} \circ \iota_{\Omega_{\Lambda^{e}}}, 0,0, p_{Y} \circ f_{w} \circ \iota_{\Omega_{\Gamma^{e}}}\right) .
$$

For $w \in I(\Gamma)$ we get

$$
\left(0, p_{X} \circ f_{w} \circ \iota_{\Omega_{\Lambda} e}, p_{\Gamma} \circ f_{w} \circ \iota_{\Omega_{\Gamma} e}, 0\right)
$$

and for $w \notin I(\Lambda) \cup I(\Gamma)$ we get

$$
\left(0, p_{X} \circ f_{w} \circ \iota_{\Omega_{\Lambda} e}, 0, p_{Y} \circ f_{w} \circ \iota_{\Omega_{\Gamma} e}\right) .
$$

The maps in the first coordinate of (7) are exactly what must be quotiented out of $\operatorname{Hom}_{\Lambda^{e}}\left(\Omega_{\Lambda^{e}}, \Lambda\right)$ to get $\mathrm{HH}^{1}(\Lambda)$. Therefore there is an exact sequence

$$
0 \rightarrow K(\Lambda, \Gamma) \rightarrow \mathrm{HH}^{1}(\Lambda \sqcup \Gamma) \stackrel{\pi}{\rightarrow} \operatorname{HH}^{1}(\Lambda) \oplus \operatorname{HH}^{1}(\Gamma) \rightarrow 0
$$

of vector spaces, where $\pi$ kills the second and fourth summands of (6), and $K(\Lambda, \Gamma)$ is defined to be the kernel of $\pi$.

$\operatorname{HH}^{1}(\Lambda \sqcup \Gamma)$ is a $\operatorname{HH}^{0}(\Lambda \sqcup \Gamma)=Z(\Lambda \sqcup \Gamma)$-module. The module structure comes from the action of the centre on homomorphisms: if $z \in Z(\Lambda \sqcup \Gamma)$ and $f$ is a $(\Lambda \sqcup \Gamma)^{e}$ homomorphism with image in $\Lambda \sqcup \Gamma$ then $z \cdot f$ is defined by $(z \cdot f)(x)=z x$. We assume from now on that $\Lambda$ and $\Gamma$ are non-trivial, so that $Z(\Lambda \sqcup \Gamma)$ contains no elements of the copies of $I(\Lambda)$ or $I(\Gamma)$ in $\Lambda \sqcup \Gamma$. It follows that (8) is an exact sequence of $Z(\Lambda \sqcup \Gamma)$ modules, with $Z(\Lambda \sqcup \Gamma)$ acting trivially on the quotient $\operatorname{HH}^{1}(\Lambda \sqcup \Gamma) / K(\Lambda, \Gamma)$.

5.2. The main theorem on Hochschild cohomology of coproducts. Let $\Lambda$ and $\Gamma$ be augmented algebras. Write $s \operatorname{HH}(\Lambda)$ for the augmented subalgebra of $\mathrm{HH}(\Lambda)$ generated by $1 \in \mathrm{HH}^{0}(\Lambda)$ and all $\operatorname{HH}^{i}(\Lambda)$ with $i>0$. Let $K$ be the augmented algebra such that $I(K)=K(\Lambda, \Gamma)$ with trivial multiplication.

Theorem 5.2. Suppose $\Lambda$ and $\Gamma$ are augmented algebras such that $\Lambda^{e}$ and $\Gamma^{e}$ are self-injective and $Z(\Lambda \sqcup \Gamma)=k$. Then there is an isomorphism of algebras

$$
\mathrm{HH}(\Lambda \sqcup \Gamma) \cong s \operatorname{HH}(\Lambda) * s \operatorname{HH}(\Gamma) * K .
$$

This is reminiscent of the results of [HS71, VI.14] on ordinary cohomology of coproducts of groups: from degree two onwards the cohomology groups are the products of the cohomology groups of the factors.

Lemma 6.16 will show that the only cases in which $Z(\Lambda \sqcup \Gamma)$ can be larger than $k$ are covered by Example 5.3.

Proof of Theorem 5.2. We begin by using dimension shifting and the EckmannShapiro lemma to get a vector space isomorphism between $\mathrm{HH}(\Lambda \sqcup \Gamma)$ and $s \mathrm{HH}(\Lambda) *$ $s \mathrm{HH}(\Gamma) * K$. They agree in degree zero by hypothesis and in degree one by the 
results of Section 5.1. For $n>1$,

(9)

$$
\begin{aligned}
& \operatorname{Ext}_{(\Lambda \sqcup \Gamma)^{e}}^{n}(\Lambda \sqcup \Gamma, \Lambda \sqcup \Gamma) \cong \operatorname{Ext}_{(\Lambda \sqcup \Gamma)^{e}}^{n-1}\left(\Omega_{(\Lambda \sqcup \Gamma)^{e}}, \Lambda \sqcup \Gamma\right) \\
& \cong \operatorname{Ext}_{(\Lambda \sqcup \Gamma)^{e}}^{n-1}\left(\Omega_{\Lambda^{e} \uparrow}(\Lambda \sqcup \Gamma)^{e}, \Lambda \sqcup \Gamma\right) \oplus \operatorname{Ext}_{(\Lambda \sqcup \Gamma)^{e}}^{n-1}\left(\Omega_{\Gamma^{e} \uparrow}(\Lambda \sqcup \Gamma)^{e}, \Lambda \sqcup \Gamma\right) \\
& \cong \operatorname{Ext}_{\Lambda^{e}}^{n-1}\left(\Omega_{\Lambda^{e}}, \Lambda \sqcup \Gamma\right) \oplus \operatorname{Ext}_{\Gamma^{e}}^{n-1}\left(\Omega_{\Gamma^{e}}, \Lambda \sqcup \Gamma\right) \\
& \cong \operatorname{Ext}_{\Lambda^{e}}^{n}(\Lambda, \Lambda \sqcup \Gamma) \oplus \operatorname{Ext}_{\Gamma^{e}}^{n}(\Gamma, \Lambda \sqcup \Gamma) \\
& \cong \operatorname{Ext}_{\Lambda^{e}}^{n}(\Lambda, \Lambda) \oplus \operatorname{Ext}_{\Gamma^{e}}^{n}(\Gamma, \Gamma)
\end{aligned}
$$

showing that $\mathrm{HH}(\Lambda \sqcup \Gamma)$ and $s \mathrm{HH}(\Lambda) * s \mathrm{HH}(\Gamma) * K$ agree as vector spaces in degree $n$. The third isomorphism uses the Eckmann-Shapiro lemma, which applies because $(\Lambda \sqcup \Gamma)^{e}$ is free on restriction to $\Lambda^{e}$ and $\Gamma^{e}$. The fifth isomorphism is because $\Lambda \sqcup \Gamma$ is isomorphic as a $\Lambda^{e}$-module to $\Lambda \oplus X$ where $X$ is free, hence injective under our hypothesis of self-injectivity.

We now need to find an isomorphism of algebras. Choose bimodule resolutions $\left(P_{*}, d_{*}^{P}\right)$ of $\Lambda$ and $\left(Q_{*}, d_{*}^{Q}\right)$ of $\Gamma$ such that $P_{0}=\Lambda^{e}$ and $Q_{0}=\Gamma^{e}$. We can then form a bimodule resolution $P * Q$ of $\Lambda \sqcup \Gamma$ :

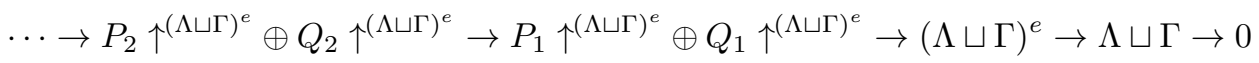

The differential of this resolution is $d_{*}^{P} \uparrow(\Lambda \sqcup \Gamma)^{e} \oplus d_{*}^{Q} \uparrow(\Lambda \sqcup \Gamma)^{e}$ in degrees at least two. At degree one it is $d_{1}^{P} \uparrow(\Lambda \sqcup \Gamma)^{e} \oplus d_{1}^{Q} \uparrow(\Lambda \sqcup \Gamma)^{e}$ regarded as a map to $\Omega_{\Lambda^{e}} \uparrow(\Lambda \sqcup \Gamma)^{e}$ $\oplus \Omega_{\Gamma e} \uparrow(\Lambda \sqcup \Gamma)^{e} \cong \Omega_{(\Lambda \sqcup \Gamma)^{e}}$, composed with the inclusion of this into $(\Lambda \sqcup \Gamma)^{e}$. At degree zero it is $x \otimes y \mapsto x y$.

Tracing through the isomorphisms of (9) as in the proof of Theorem 4.1 we find that they provide an injection $j_{\Lambda}: \mathrm{HH}^{\geq 1}(\Lambda) \hookrightarrow \mathrm{HH}(\Lambda \sqcup \Gamma)$ that sends the cohomology class of a chain map $\alpha_{*}$ on $P_{*}$ to the class of $\alpha_{*} \uparrow(\Lambda \sqcup \Gamma)^{e}$ regarded as a map on $P * Q$ killing the $Q_{*} \uparrow(\Lambda \sqcup \Gamma)^{e}$ summands. This is multiplicative, because

$$
\left(\alpha_{*} \circ \beta_{*}\right) \uparrow^{(\Lambda \sqcup \Gamma)^{e}}=\alpha_{*} \uparrow^{(\Lambda \sqcup \Gamma)^{e}} \circ \beta_{*} \uparrow^{(\Lambda \sqcup \Gamma)^{e}} .
$$

There is a similar injection $j_{\Gamma}: \mathrm{HH}^{\geq 1}(\Gamma) \hookrightarrow \mathrm{HH}(\Lambda \sqcup \Gamma)$, and the product of elements of $\operatorname{im} j_{\Lambda}$ and $\operatorname{im} j_{\Gamma}$ is zero because of the direct sum decomposition of $P * Q$ in degrees at least one.

It only remains to show that an element of $K(\Lambda, \Gamma)$ has product zero with any other element $x$ of positive degree. Because Hochschild cohomology is graded commutative it suffices to show $k \cdot x=0$. The product $k \cdot x$ is represented by a cocycle of degree at least two with image contained in $\bigoplus_{i \geq 2} R_{i}$, but these are exactly the cocycles shown to be zero in cohomology by the last isomorphism of (9).

Example 5.3. Let $\Lambda=k[x] / x^{2}$ and $\Gamma=k[y] / y^{2}$, where $k$ has characteristic not two. $\mathrm{HH}(\Lambda)$ is the graded commutative ring $k\left[x_{0}, x_{1}, x_{2}\right] /\left(x_{0}^{2}, x_{1}^{2}, x_{0} x_{1}, x_{0} x_{2}\right)$, where degrees are given by subscripts. We have $Z(\Lambda \sqcup \Gamma)=k[x y+y x]$, and computing as in the proof of Theorem 5.2 the algebra structure of $\mathrm{HH}(\Lambda \sqcup \Gamma)$ is given by

$$
s \mathrm{HH}(\Lambda) * s \mathrm{HH}(\Gamma) * Z(\Lambda \sqcup \Gamma) * K
$$

where the augmentations on $s \mathrm{HH}(\Lambda)$ and $s \mathrm{HH}(\Gamma)$ kill elements of positive degree, and $K$ is the augmented algebra with trivial multiplication whose augmentation ideal is the subquotient of $(\Lambda \sqcup \Gamma) \oplus(\Lambda \sqcup \Gamma)$ given by

$$
\frac{\langle(x y x, 0),(x y x y x, 0),(x y x y x y x, 0) \ldots\rangle+\langle(0, y x y),(0, y x y x y),(0, y x y x y x y) \ldots\rangle}{\langle(x y x \cdots y x,-y x y \cdots x y)\rangle} .
$$

$Z(\Lambda \sqcup \Gamma)$ lies in degree zero and $I(K)$ lies in degree one. 


\section{Cohomology of products}

We begin our investigation of the cohomology of a product in Section 6.1 using bimodule resolutions for algebras $\Lambda$ and $\Gamma$ to build a bimodule resolution of $\Lambda * \Gamma$. In Section 6.2 we use this resolution to study the ordinary cohomology $E(\Lambda * \Gamma)$ by applying the functor $-\otimes_{\Lambda * \Gamma} k$, and prove in Theorem 6.9 that $E(\Lambda * \Gamma)=$ $E(\Lambda) \sqcup E(\Gamma)$, so long as $\Lambda$ and $\Gamma$ are either finite-dimensional or finitely generated and graded connected, that is, positively graded with zero degree part equal to $k$. Finally in Section 6.3 we return to our bimodule resolution to investigate Hochschild cohomology of products, showing that $\mathrm{HH}(\Lambda * \Gamma)$ decomposes, modulo a certain ideal, into a direct sum of terms coming from the cohomology of $\Lambda$ and $\Gamma$.

The reason for the restriction to finite-dimensional or finitely generated graded connected algebras is that we require the existence of a bimodule resolution $\left(P_{*}, d_{*}\right)$ of $\Lambda$ with the property that

$$
\operatorname{im} d_{*} \subseteq I(\Lambda) \cdot P_{*}+P_{*} \cdot I(\Lambda)
$$

(and a similar resolution for $\Gamma$ ). Such resolutions exist given our restriction on $\Lambda$ and $\Gamma$ : if they are finite dimensional then a minimal projective resolution exists which certainly has this property. If $\Lambda$ is graded with $\Lambda_{0}=k$ and and $M$ is a finitely generated graded $\Lambda$-module with $\left\{m_{1} \ldots m_{r}\right\}$ a minimal homogeneous generating set then the kernel of the map $\bigoplus \Lambda e_{i} \rightarrow M$ given by $e_{i} \mapsto m_{i}$ is concentrated in positive degree. To see this, let $\sum \lambda_{i} m_{i}=0$ and suppose that $\lambda_{1}$ has a non-zero degree zero component, which we may take to be 1 . Let the degree of $m_{1}$ be $N$ and project onto the degree $N$ component of $\sum \lambda_{i} m_{i}=0$. We get

$$
m_{1}+\sum_{i>1}\left[\lambda_{i}\right]_{N-\operatorname{deg}\left(m_{i}\right)} m_{i}=0
$$

where $[\lambda]_{m}$ denotes the degree $m$ component of $\lambda \in \Lambda$. It follows that $m_{1}$ was not essential in the generating set. Using this repeatedly for $\Lambda^{e}$ we can build a bimodule resolution with the property (10). In general however, no such resolution exists.

Example 6.1. Let $\Lambda=k[x, y] /\left(x^{2}+y^{2}-x\right)$, augmented by $\epsilon_{\Lambda}(x)=\epsilon_{\Lambda}(y)=0$. Then $I(\Lambda)=(x, y)$ is a principal ideal if and only if $k$ contains a square root $i$ of -1 , in which case $I(\Lambda)=(y+i x)$ and there is a projective resolution

$$
0 \rightarrow \Lambda \stackrel{y+i x}{\rightarrow} \Lambda \stackrel{\varepsilon}{\rightarrow} k \rightarrow 0 .
$$

If $k$ has no square root of -1 , any projective resolution

$$
\cdots \rightarrow Q_{1} \stackrel{d_{1}}{\rightarrow} \Lambda \rightarrow k \rightarrow 0
$$

has at least two summands at degree one ( $\Lambda$ is indecomposable as a left module over itself as it is an integral domain, so the only finitely generated projectives are free). There is no projective resolution $\left(Q_{*}, d_{*}\right)$ in which ker $d_{1} \subseteq I(\Lambda) \cdot Q_{1}$ : if there were $\operatorname{dim} \operatorname{Ext}_{\Lambda}^{1}(k, k)$ would be equal both to $\operatorname{dim} \operatorname{Hom}_{\Lambda}\left(Q_{1}, k\right)>1$ and to $\operatorname{dim} \operatorname{Hom}_{\Lambda}(I(\Lambda), k)=1$. It follows that there is no bimodule resolution $\left(P_{*}, d_{*}\right)$ of $\Lambda$ satisfying (10), for if there were we could obtain a projective resolution $\left(Q_{*}, \delta_{*}\right)$ of $k$ in which $\operatorname{ker} \delta_{1} \subseteq I(\Lambda) \cdot Q_{1}$ by applying $-\otimes_{\Lambda} k$.

$A$ projective resolution of $k$ in the case where the field has no square root of -1 is given by letting every term after the zeroth be $\Lambda \oplus \Lambda$ with differentials alternating between

$$
\left(\begin{array}{cc}
y & x \\
1-x & y
\end{array}\right) \text { and }\left(\begin{array}{cc}
-y & 1-x \\
x & y
\end{array}\right)
$$


6.1. Bimodule resolutions of products. Let $\Lambda$ and $\Gamma$ be augmented algebras such that there exist bimodule resolutions $\left(P_{*}, d_{*}^{P}\right)$ and $\left(Q_{*}, d_{*}^{Q}\right)$ of $\Lambda$ and $\Gamma$ with the 'smallness' property (10). Furthermore, assume $P_{0}=\Lambda^{e}, Q_{0}=\Gamma^{e}$, and $d_{0}^{P}(x \otimes y)=$ $x y$ with $d_{0}^{Q}$ defined in the same way. Our goal in this section is to produce a bimodule resolution $\left((P \sqcup Q)_{*}, \delta_{*}\right)$ for $\Lambda * \Gamma$ with the same smallness property.

We use a bar to denote an induction functor $\uparrow(\Lambda * \Gamma)^{e}$, and will write $d$ instead of $d^{P}$ or $d^{Q}$ — which subalgebra we induce from, and which differential we are applying, should be clear by the context. Given bimodules $A$ and $B$ for an augmented algebra $\Delta$ we write $A \hat{\otimes} B$ for $A \otimes_{\Delta} k \otimes_{\Delta} B$. If $A$ and $B$ are projective then so is $A \hat{\otimes} B$; this follows because $\Delta^{e} \hat{\otimes} \Delta^{e} \cong \Delta^{e}$.

We will need the following technical lemma when we build the resolution $P \sqcup Q$.

Lemma 6.2. Let $\Lambda$ and $\Gamma$ be augmented algebras, $M$ a $\Lambda$-bimodule, $N$ be a $\Gamma$ bimodule and $V$ be $a \Lambda * \Gamma$-bimodule. Let $f$ be a $\Lambda$-bimodule map $M \rightarrow \Lambda^{e}$ with image contained in $I(\Lambda) \otimes \Lambda+\Lambda \otimes I(\Lambda)$. Then

$$
\mathcal{L}(f): M \uparrow^{\Lambda * \Gamma} \hat{\otimes} N \uparrow^{\Lambda * \Gamma} \hat{\otimes} V \rightarrow N \uparrow \Lambda * \Gamma \hat{\otimes} V
$$

$\left(x \otimes_{\Lambda}\left(y \otimes_{k} \lambda+\lambda^{\prime} \otimes_{k} y^{\prime}\right) \otimes_{\Lambda} z\right) \hat{\otimes}\left(w \otimes_{\Gamma} n \otimes u\right) \hat{\otimes} v \mapsto\left(\epsilon_{\Lambda * \Gamma}(\lambda z w) x y \otimes_{\Gamma} n \otimes_{\Gamma} u\right) \hat{\otimes} v$

where $x, z, w, u \in \Lambda * \Gamma, y, y^{\prime} \in I(\Lambda), \lambda, \lambda^{\prime} \in \Lambda, n \in N, v \in V$, is a well-defined $\Lambda * \Gamma$-bimodule map.

Proof. The $\lambda^{\prime} \otimes y^{\prime}$ term can be ignored since we can move $y^{\prime}$ through $\otimes_{\Lambda}$ then move $y^{\prime} z$ through $\hat{\otimes}$ to get zero. Checking well-definedness is routine except at the first $\otimes_{\Gamma}$. For $\gamma \in \Gamma$ we have

$$
\begin{aligned}
& \left(x \otimes_{\Lambda}\left(y \otimes_{k} \lambda\right) \otimes_{\Lambda} z\right) \hat{\otimes}\left(w \otimes_{\Gamma} \gamma n \otimes u\right) \hat{\otimes} v \mapsto\left(\epsilon_{\Lambda * \Gamma}(\lambda z w) x y \otimes_{\Gamma} \gamma n \otimes_{\Gamma} u\right) \hat{\otimes} v \\
& \left(x \otimes_{\Lambda}\left(y \otimes_{k} \lambda\right) \otimes_{\Lambda} z\right) \hat{\otimes}\left(w \gamma \otimes_{\Gamma} n \otimes u\right) \hat{\otimes} v \mapsto\left(\epsilon_{\Lambda * \Gamma}(\lambda z w \gamma) x y \otimes_{\Gamma} n \otimes_{\Gamma} u\right) \hat{\otimes} v .
\end{aligned}
$$

The two images are equal because for $y \in I(\Lambda)$ we have $y \gamma=\epsilon_{\Lambda * \Gamma}(\gamma) y$.

There is a right-handed version of this lemma giving a map

$$
\mathcal{R}(f): V \hat{\otimes} N \uparrow^{\Lambda * \Gamma} \hat{\otimes} M \uparrow \Lambda * \Gamma \rightarrow V \hat{\otimes} N \uparrow \Lambda * \Gamma
$$

under the same hypotheses.

Definition 6.3. $(P \sqcup Q)_{n}$ is defined to be $(\Lambda * \Gamma)^{e}$ if $n=0$, and

$$
\bigoplus \cdots \hat{\otimes} \bar{P}_{i_{r-1}} \hat{\otimes} \bar{Q}_{i_{r}} \hat{\otimes} \bar{P}_{i_{r+1}} \hat{\otimes} \cdots
$$

if $n>0$, where the direct sum is over all tuples $\left(i_{1}, i_{2}, \ldots\right)$ of strictly positive integers such that $\sum_{j} i_{j}=n$.

We now need to define the differential $\delta_{n}$ on $(P \sqcup Q)_{n}$, which is spanned by elements of the form

$$
a_{1} \hat{\otimes} a_{2} \hat{\otimes} \cdots \hat{\otimes} a_{N}
$$

where the $a_{i}$ alternate between elements of $\bar{P}_{*}$ and $\bar{Q}_{*}$, and the sum of the degrees of the $a_{i}$ is $n$ (we say $a_{i}$ has degree $m$ if $a_{i} \in \bar{P}_{m} \cup \bar{Q}_{m}$ ). Roughly, $\delta$ will be a sum of two terms, one using $\bar{d}^{P}$ or $\bar{d}^{Q}$ to drop the degree of $a_{1}$ by one, the other doing the same to $a_{N}$.

Definition 6.4. Let $a$ be an element of the form (11) with $N>1$. If $a_{1}$ has degree greater than one we define

$$
l(a)=\bar{d}\left(a_{1}\right) \hat{\otimes} a_{2} \cdots \hat{\otimes} a_{N} .
$$

If $a_{1}$ lies in $\bar{P}_{1}$ or $\bar{Q}_{1}$ then we define

$$
l(a)=\mathcal{L}\left(d_{1}\right)\left(a_{1} \hat{\otimes} a_{2} \cdots \hat{\otimes} a_{N}\right) .
$$


Here we used the hypothesis that $d^{P}$ and $d^{Q}$ satisfy (10), at least at degree one: without this $\mathcal{L}(d)_{1}$ would not be defined.

Definition 6.5. Let $a$ be an element of the form (11) with $N>1$. If $a_{N}$ has degree greater than one we define

$$
r(a)=a_{1} \hat{\otimes} a_{2} \cdots \hat{\otimes} \bar{d}\left(a_{N}\right) .
$$

If $a_{N}$ lies in $\bar{P}_{1}$ or $\bar{Q}_{1}$ then we define

$$
r(a)=\mathcal{R}\left(d_{1}\right)\left(a_{1} \hat{\otimes} a_{2} \cdots \hat{\otimes} a_{N}\right) .
$$

Definition 6.6. The differential $\delta$ on $P \sqcup Q$ is defined as follows. $\delta_{0}:(\Lambda * \Gamma)^{e} \rightarrow$ $\Lambda * \Gamma$ is $x \otimes y \mapsto x y$. On an element a of the form (11) with $N=1$ we define $\delta_{*}(a)=\bar{d}_{*}(a)$. On an element a of the form (11) with $N>1$, lying in $(P \sqcup Q)_{n}$, we define

$$
\delta_{n}(a)=l(a)+(-1)^{n} r(a)
$$

This means that if $r, s>1$ then $\delta$ maps

$$
\bar{P}_{r} \hat{\otimes} \cdots \hat{\otimes} \bar{Q}_{s} \rightarrow \bar{P}_{r-1} \hat{\otimes} \cdots \hat{\otimes} \bar{Q}_{s} \oplus \bar{P}_{r} \hat{\otimes} \cdots \hat{\otimes} \bar{Q}_{s-1} .
$$

Note also that $\left(\bar{P}_{*}, \bar{d}_{*}\right)$ and $\left(\bar{Q}_{*}, \bar{d}_{*}\right)$ are subcomplexes of $P \sqcup Q$.

Example 6.7. Suppose $p \in P_{1}$ and $q \in Q_{1}$ are such that $d_{1}^{P}(p)=1 \otimes \lambda-\lambda \otimes 1$ for $\lambda \in I(\Lambda)$ and $d_{1}^{Q}(q)=1 \otimes \gamma-\gamma \otimes 1$ for $\gamma \in I(\Gamma)$. The first few terms of $P \sqcup Q$ are

$$
\cdots \rightarrow \bar{P}_{2} \oplus \bar{P}_{1} \hat{\otimes} \bar{Q}_{1} \oplus \overline{Q_{1}} \hat{\otimes} \bar{P}_{1} \oplus \bar{Q}_{2} \rightarrow \bar{P}_{1} \oplus \bar{Q}_{1} \rightarrow(\Lambda * \Gamma)^{e} \rightarrow \Lambda * \Gamma \rightarrow 0 .
$$

Identifying $\bar{P}_{1} \hat{\otimes} \bar{Q}_{1}$ with

$$
(\Lambda * \Gamma) \otimes_{\Lambda} P_{1} \otimes_{\Lambda} k \otimes_{\Gamma} Q_{1} \otimes_{\Gamma}(\Lambda * \Gamma)
$$

we have

$$
\delta_{1}\left(1 \otimes_{\Lambda} p \otimes_{\Lambda} 1 \otimes_{\Gamma} q \otimes_{\Gamma} 1\right)=-\lambda \otimes_{\Gamma} q \otimes_{\Gamma} 1+1 \otimes_{\Lambda} p \otimes \gamma .
$$

Lemma 6.8. $\left((P \sqcup Q)_{*}, \delta_{*}\right)$ is a bimodule resolution of $\Lambda * \Gamma$ satisfying (10).

Proof. Checking that $\delta^{2}=0$ is straightforward, using the property (10). That $\left((P \sqcup Q)_{*}, \delta_{*}\right)$ satisfies (10) itself is immediate from the definition of $\delta_{*}$. To show the complex is exact we write down a contracting homotopy $\sigma$. Choose contracting homotopies $s_{*}, t_{*}$ for $\left(P_{*}, d_{*}\right)$ and $\left(Q_{*}, d_{*}\right)$ which are homomorphisms of left $\Lambda$ and $\Gamma$-modules respectively. Then $s_{*}$ consists of a family of left $\Lambda$-module maps $s_{i}: P_{i} \rightarrow P_{i+1}$ for $i \geq 0$ and $s_{-1}: \Lambda \rightarrow P_{0}=\Lambda^{e}$ such that

$$
s_{r} d_{r+1}+d_{r+2} s_{r+1}=\operatorname{id}_{P_{r+1}} \quad r \geq-1
$$

and $d_{0} s_{-1}=\operatorname{id}_{\Lambda}$. We insist that $s_{-1}(\lambda)=\lambda \otimes 1$ and $t_{-1}(\gamma)=\gamma \otimes 1$. We use $s_{*}$ and $t_{*}$ to build a contracting homotopy $\sigma_{*}$ for $P \sqcup Q$ which is a homomorphism of left $\Lambda * \Gamma$-modules.

Firstly, define $\sigma_{-1}(x)=x \otimes 1$ for $x \in \Lambda * \Gamma$, and let $\sigma_{0}$ be the map of left $\Lambda * \Gamma$-modules $(\Lambda * \Gamma)^{e} \rightarrow(P \sqcup Q)_{1}$ such that:

$$
\begin{aligned}
& \sigma_{0}(1 \otimes 1)=0 \\
& \sigma_{0}(1 \otimes \lambda)=1 \otimes s_{0}(\lambda) \otimes 1 \in \bar{P}_{1} \\
& \sigma_{0}(1 \otimes \gamma)=1 \otimes t_{0}(\gamma) \otimes 1 \in \bar{Q}_{1}
\end{aligned}
$$

for $\lambda \in I(\Lambda)$ and $\gamma \in I(\Gamma)$.

Now suppose that

$$
a=a_{1} \hat{\otimes} \cdots \hat{\otimes} a_{N-1} \hat{\otimes}\left(1 \otimes_{\Lambda} p \otimes_{\Lambda}(\alpha+\gamma)\right) \in\left(\cdots \hat{\otimes} \bar{P}_{m}\right) \subset(P \sqcup Q)_{n}
$$


where $p \in P_{m}, \alpha \in k$ and $\gamma \in I(\Gamma)$. Noting that $t_{0}(1 \otimes \gamma) \in Q_{1}$ and $s_{m}(p) \in P_{m+1}$, define

$$
\begin{aligned}
\sigma_{n}(a) & =(-1)^{n+1} a_{1} \hat{\otimes} \cdots \hat{\otimes} a_{N-1} \hat{\otimes}\left(1 \otimes_{\Lambda} s_{m}(p) \otimes_{\Lambda} \alpha\right) \\
& +(-1)^{n+1} a_{1} \hat{\otimes} \cdots \hat{\otimes}\left(1 \otimes_{\Lambda} p \otimes_{\Lambda} 1\right) \hat{\otimes}\left(1 \otimes_{\Gamma} t_{0}(1 \otimes \gamma) \otimes_{\Gamma} 1\right) .
\end{aligned}
$$

in $\left(\cdots \hat{\otimes} \bar{P}_{m+1}\right) \oplus\left(\cdots \hat{\otimes} \bar{P}_{m} \hat{\otimes} \bar{Q}_{1}\right)$. A similar definition is made on summands of $(P \sqcup Q)_{n}$ ending in a term from $\bar{Q}$. The reader may verify that $\sigma_{*}$ is a contracting homotopy for $\left((P \sqcup Q)_{*}, \delta_{*}\right)$.

6.2. Ordinary cohomology of products. We now use this bimodule resolution to study the ordinary cohomology of a product $\Lambda * \Gamma$ with the aim of proving the following theorem.

Theorem 6.9. Let $\Lambda$ and $\Gamma$ be augmented $k$-algebras which are either finitedimensional or graded connected and finitely generated. Then there is an isomorphism of algebras

$$
\operatorname{Ext}_{\Lambda * \Gamma}^{*}(k, k) \cong \operatorname{Ext}_{\Lambda}^{*}(k, k) \sqcup \operatorname{Ext}_{\Gamma}^{*}(k, k) .
$$

This result generalises the one for local $k$-algebras appears in [Moo09], and for finitely generated graded connected algebras in [PP05, Proposition 1.1].

Define

$$
\left(R_{*}, \partial_{*}\right)=\left((P \sqcup Q)_{*} \otimes_{\Lambda * \Gamma} k, \delta_{*} \otimes_{\Lambda * \Gamma} k\right) .
$$

Then $H_{n}\left(R_{*}, \partial_{*}\right)$ computes $\operatorname{Tor}_{n}^{\Lambda * \Gamma}(\Lambda * \Gamma, k)$ which is zero for $n>0$ as $\Lambda * \Gamma$ is projective, hence flat, as a right module over itself. It follows that $\left(R_{*}, \partial_{*}\right)$ is a projective resolution of $k$ as a left $\Lambda * \Gamma$-module. Because

$$
\operatorname{im} \delta_{*} \subseteq I(\Lambda * \Gamma) \cdot(P \sqcup Q)_{*}+(P \sqcup Q)_{*} \cdot I(\Lambda * \Gamma)
$$

we have $\operatorname{im} \partial_{*} \subseteq I(\Lambda * \Gamma) \cdot R_{*}$.

A resolution like $R_{*}$ appears in [Moo09].

Lemma 6.10. Let $\left(R_{*}, \partial_{*}\right)$ be a projective resolution of the trivial module over an augmented algebra $\Delta$ such that $\operatorname{im} \partial_{*} \subseteq I(\Delta) \cdot R_{*}$. Then

$$
\operatorname{Ext}_{\Delta}^{n}(k, k) \cong \operatorname{Hom}_{\Delta}\left(R_{n}, k\right) .
$$

Proof. The differential induced by $\partial_{*}$ on the cocomplex

$$
\operatorname{Hom}_{\Delta}\left(R_{0}, k\right) \rightarrow \operatorname{Hom}_{\Delta}\left(R_{1}, k\right) \rightarrow \operatorname{Hom}_{\Delta}\left(R_{2}, k\right) \rightarrow \cdots
$$

is zero, because any $\Delta$-homomorphism $R_{n} \rightarrow k$ kills $I(\Delta) \cdot R_{n}$. The $n$th cohomology of this cocomplex computes $\operatorname{Ext}_{\Delta}^{n}(k, k)$, so the result follows.

Similarly, $\left(\mathrm{P}_{*}, \mathfrak{d}_{*}^{P}\right)=\left(P_{*} \otimes_{\Lambda} k, d_{*}^{P} \otimes_{\Lambda} k\right)$ and $\left(\mathrm{Q}_{*}, \mathfrak{d}_{*}^{Q}\right)=\left(Q_{*} \otimes_{\Lambda} k, d_{*}^{Q} \otimes_{\Lambda} k\right)$ are projective resolutions of $k$ such that

$$
\begin{aligned}
\operatorname{Ext}_{\Lambda}^{n}(k, k) & \cong \operatorname{Hom}_{\Lambda}\left(\mathrm{P}_{n}, k\right) \\
& \cong \operatorname{Hom}_{\Lambda}\left(P_{n} \otimes_{\Lambda} k, k\right) \\
& \cong \operatorname{Hom}_{k}\left(k \otimes_{\Lambda} P_{n} \otimes_{\Lambda} k, k\right)
\end{aligned}
$$

with an analogous result for the cohomology of $\Gamma$.

Lemma 6.11. $\operatorname{Ext}_{\Lambda * \Gamma}^{n}(k, k)$ is isomorphic as a k-vector space to the degree $n$ part of $E(\Lambda) \sqcup E(\Gamma)$. 
Proof. Our restrictions on $\Lambda$ and $\Gamma$ are enough to guarantee that $P_{n}$ is finitelygenerated, so $\operatorname{Hom}_{k}\left(k \otimes_{\Lambda} P_{n} \otimes_{\Lambda} k, k\right) \cong k \otimes_{\Lambda} P_{n} \otimes_{\Lambda} k$ as a vector space. Writing $D$ for $\operatorname{Hom}_{k}(-, k)$, a typical summand of the dual of the $n$th degree part of $E(\Lambda) \sqcup E(\Gamma)$ looks like

$$
\begin{gathered}
\cdots \otimes_{k} D E^{i_{r-1}}(\Lambda) \otimes_{k} D E^{i_{r}}(\Gamma) \otimes_{k} D E^{i_{r+1}}(\Lambda) \otimes_{k} \cdots \\
\cong \cdots \otimes_{\Gamma} k \otimes_{\Lambda} P_{i_{r-1}} \otimes_{\Lambda} k \otimes_{\Gamma} Q_{i_{r}} \otimes_{\Gamma} k \otimes_{\Lambda} P_{i_{r+1}} \otimes_{\Lambda} k \otimes_{\Gamma} \cdots
\end{gathered}
$$

The $k$-dual of $E^{n}(\Lambda * \Gamma)$ is $k \otimes_{\Lambda * \Gamma}(P \sqcup Q)_{n} \otimes_{\Lambda * \Gamma} k=k \otimes_{\Lambda * \Gamma} R_{n}$, a typical summand of which is

$$
\begin{aligned}
& \cdots \otimes_{\Lambda * \Gamma} \bar{P}_{i_{r-1}} \otimes_{\Lambda * \Gamma} k \otimes_{\Lambda * \Gamma} \bar{Q}_{i_{r}} \otimes_{\Lambda * \Gamma} k \otimes_{\Lambda * \Gamma} \bar{P}_{i_{r+1}} \otimes_{\Lambda * \Gamma} k \otimes_{\Lambda * \Gamma} \cdots \\
\cong & \cdots \otimes_{\Gamma} k \otimes_{\Lambda} P_{i_{r-1}} \otimes_{\Lambda} k \otimes_{\Gamma} Q_{i_{r}} \otimes_{\Gamma} k \otimes_{\Lambda} P_{i_{r+1}} \otimes_{\Lambda} k \otimes_{\Gamma} \cdots
\end{aligned}
$$

The result follows.

We now look at how $E\left(p_{\Lambda}\right)$ and $E\left(p_{\Gamma}\right)$ behave on the level of chain maps. Consider a chain map $f_{*}: \mathrm{P}_{*} \rightarrow \mathrm{P}_{*}$ of degree $n>0$, consisting of a family of maps $f_{i}: \mathrm{P}_{i+n} \rightarrow \mathrm{P}_{i}$ for $i \geq 0$, with $\mathrm{P}_{0}=\Lambda^{e} \otimes_{\Lambda} k$ identified with $\Lambda$ so that $f_{0}: \mathrm{P}_{n} \rightarrow \Lambda$. We claim that $E\left(p_{\Lambda}\right)$ applied to the cohomology element represented by $f_{*}$ can be represented by a chain map $F(f)_{*}$ looking like id $\otimes f_{*}$ where this makes sense, and zero elsewhere.

In defining $F(f)_{*}$ we will identify a summand of $R_{*}$ ending with a term from $P_{*}$ with:

$$
\cdots \otimes_{\Lambda} k \otimes_{\Gamma} Q_{r} \otimes_{\Gamma} k \otimes_{\Lambda} P_{m} \otimes_{\Lambda} k .
$$

Definition 6.12. $F(f)_{*}$ is the chain map on $R_{*}$ defined as follows.

- $F(f)_{*}$ acts as zero on any summand of $R_{i+n}$ not ending with $P_{m}$ for $m \geq n$.

- On a summand of $R_{i+n}$ of the form (13) with $m>n, F(f)_{i}$ maps

$$
\cdots \otimes_{\Lambda} 1 \otimes_{\Gamma} q \otimes_{\Gamma} 1 \otimes_{\Lambda} p \otimes_{\Lambda} 1 \mapsto \cdots \otimes_{\Lambda} 1 \otimes_{\Gamma} q \otimes_{\Gamma} 1 \otimes f\left(p \otimes_{\Lambda} 1\right) .
$$

- On a summand of $R_{i+n}$ of the form (13) with $m=n, F(f)_{i}$ maps

$$
\begin{aligned}
& \cdots \otimes_{\Lambda} 1 \otimes_{\Gamma} q \otimes_{\Gamma} 1 \otimes_{\Lambda} p \otimes_{\Lambda} 1 \mapsto \cdots \otimes_{\Lambda} 1 \otimes_{\Gamma} q \otimes_{\Gamma} \epsilon_{\Lambda}\left(f_{0}\left(p \otimes_{\Lambda} 1\right)\right) \\
& \text { in } \cdots \otimes_{\Lambda} k \otimes_{\Gamma} Q_{r} \otimes_{\Gamma} k \subset R_{i} .
\end{aligned}
$$

Lemma 6.13. $F(f)_{*}$ is a chain map representing the image of the cohomology class of $f_{*}$ under $E\left(p_{\Lambda}\right)$.

Proof. It is straightforward to check that $F(f)_{*}$ is a chain map. The cocycle corresponding to $F(f)_{*}$ is a map $R_{n} \rightarrow k$ that kills every summand except $\bar{P}_{n} \otimes_{\Lambda} k$. Identifying such a summand with $(\Lambda * \Gamma) \otimes_{\Lambda} P_{n} \otimes_{\Lambda} k$, the cocycle sends

$$
x \otimes p \otimes 1 \mapsto \epsilon_{\Lambda * \Gamma}(x) \epsilon_{\Lambda}\left(f_{0}(p \otimes 1)\right) .
$$

To compute $E\left(p_{\Lambda}\right)$ we need a chain map

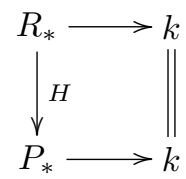

as in (2). We take $H$ to be $p_{\Lambda} \otimes k$ in degree zero, and in positive degrees to kill every summand not of the form $\bar{P}_{n} \otimes_{\Lambda} k$. On these summands, identified with $(\Lambda * \Gamma) \otimes_{\Lambda} P_{n} \otimes_{\Lambda} k, H$ should map

$$
x \otimes_{\Lambda} \otimes p \otimes 1 \rightarrow p_{\Lambda}(x) \cdot p \otimes_{\Lambda} 1 \in P_{n} \otimes_{\Lambda} k .
$$


Therefore the cocycle representing $E\left(p_{\Lambda}\right)$ applied to the cohomology element represented by $f_{*}$ is zero except on the summand $\bar{P}_{n} \otimes_{\Lambda} k=(\Lambda * \Gamma) \otimes_{\Lambda} P_{n} \otimes_{\Lambda} k$ of $R_{n}$. There it maps

$$
x \otimes p \otimes 1 \mapsto \epsilon_{\Lambda}\left(p_{\Lambda}(x) \cdot f_{0}\left(p \otimes_{\Lambda} 1\right)\right)
$$

Since $p_{\Lambda}$ is augmentation-preserving, this agrees with the cocycle for $F(f)_{*}$ above.

We now complete the proof of Theorem 6.9. Using Lemma 6.11, it is enough to prove that the morphism of augmented algebras $E\left(p_{\Lambda}\right) \sqcup E\left(p_{\Gamma}\right): E(\Lambda) \sqcup E(\Gamma) \rightarrow$ $E(\Lambda * \Gamma)$ arising from $E\left(p_{\Lambda}\right)$ and $E\left(p_{\Gamma}\right)$ is onto.

Pick a word $l_{1} g_{1} l_{2} g_{2} \cdots$ in $E(\Lambda) \sqcup E(\Gamma)$, where $l_{i} \in E(\Lambda), g_{i} \in E(\Gamma)$, let $l_{i}$ be represented by a chain map $f_{*}^{i}$ and $g_{i}$ by a chain map $f_{*}^{\prime i}$, and let $F_{*}^{i}$ and $F_{*}^{\prime i}$ be the chain maps representing $E\left(p_{\Lambda}\right)\left(l_{i}\right)$ and $E\left(p_{\Gamma}\right)\left(g_{i}\right)$ manufactured as above. Then $E\left(p_{\Lambda}\right) \sqcup E\left(p_{\Gamma}\right)$ sends $l_{1} g_{1} l_{2} g_{2} \cdots$ to the element of $E(\Lambda * \Gamma)$ represented by the composition $F_{*}^{1} \circ F_{*}^{\prime 1} \circ F_{*}^{2} \circ F_{*}^{\prime 2} \circ \cdots$.

(12) shows that $E^{n}(\Lambda * \Gamma)$ is spanned by elements that can be written as a tensor products $\alpha_{1} \otimes_{k} \beta_{1} \otimes_{k} \alpha_{2} \otimes \cdots$ where each $\alpha_{i}$ lies in $\operatorname{Hom}_{k}\left(k \otimes_{\Lambda} P_{r} \otimes_{k}, k\right) \cong E^{r}(\Lambda)$ and each $\beta_{i}$ lies in $\operatorname{Hom}_{k}\left(k \otimes_{\Lambda} Q_{s} \otimes_{k}, k\right) \cong E^{s}(\Gamma)$ for some $r, s$ depending on $i$. Let $l_{i}$ be the element of $E^{r}(\Lambda)$ corresponding to $\alpha_{i}$, and $g_{i}$ be the element of $E^{s}(\Gamma)$ corresponding to $\beta_{i}$. Lifting each $\alpha_{i}$ to a chain map $a_{i}$ on $\mathrm{P}$, and each $\beta_{i}$ to a chain map $b_{i}$ on $\mathrm{Q}$, then taking chain maps $A_{*}^{i}$ and $B_{*}^{i}$ representing $E\left(p_{\Lambda}\right)\left(l_{i}\right)$ and $E\left(p_{\Gamma}\right)\left(g_{i}\right)$, we have that $A_{*}^{1} \circ B_{*}^{1} \circ A_{*}^{2} \circ \cdots$ is a chain map that lifts $\alpha_{1} \otimes_{k} \beta_{1} \otimes_{k} \alpha_{2} \otimes \cdots$. We have realised $\alpha_{1} \otimes_{k} \beta_{1} \otimes_{k} \alpha_{2} \otimes \cdots$ as an element of $\operatorname{im} E\left(p_{\Lambda}\right) \sqcup E\left(p_{\Gamma}\right)$, finishing the proof.

6.3. Hochschild cohomology of products. We prove two main results on the structure of the Hochschild cohomology of a product. Firstly:

Proposition 6.14. Let $\Lambda$ and $\Gamma$ be non-trivial augmented algebras which are either finite-dimensional or finitely generated graded connected. Suppose that not both $E(\Lambda)$ and $E(\Gamma)$ are isomorphic to $k[x] / x^{2}$ as ungraded rings. Then $\phi_{k}: \operatorname{HH}(\Lambda * \Gamma) \rightarrow$ $E(\Lambda * \Gamma)$ is zero in positive degrees, and if $I(\Lambda)^{N}=I(\Gamma)^{N}=0$ then every element of positive degree in $\mathrm{HH}(\Lambda * \Gamma)$ has $N$ th power zero.

By Koszul duality, if $E(\Lambda) \cong k[x] / x^{2}$ then $\Lambda \cong k[x]$.

Our second result determines the structure of a certain quotient of $\operatorname{HH}(\Lambda * \Gamma)$ in terms of the cohomology of $\Lambda$ and $\Gamma$. Let $\mathrm{iHH}(\Lambda)$ be the unital subalgebra generated by the ideal $\operatorname{ker} \phi_{k}: \mathrm{HH}(\Lambda) \rightarrow E(\Lambda)$. This is naturally an augmented algebra, so we can form $\operatorname{iHH}(\Lambda) * \mathrm{iHH}(\Gamma)$. Let $A(\Gamma)$ be the annihilating ideal $\{\gamma \in \Gamma: \gamma I(\Gamma)=I(\Gamma) \gamma=0\}$.

Theorem 6.15. Let $\Lambda$ and $\Gamma$ be finite-dimensional or finitely generated graded connected algebras. Then there is an ideal $J$ of $\mathrm{HH}(\Lambda * \Gamma)$, equal to the image of the map $\pi^{*}$ appearing in (14), such that

$$
\mathrm{HH}(\Lambda * \Gamma) / J \cong(\mathrm{iHH}(\Lambda) * \mathrm{iHH}(\Gamma)) \oplus\left(E(\Lambda) \otimes_{k} A(\Gamma)\right) \oplus\left(E(\Gamma) \otimes_{k} A(\Lambda)\right)
$$

as algebras. The product of any two elements of the copy of $E(\Lambda) \otimes_{k} A(\Gamma)$ is zero in this quotient, as is the product of any two elements of $E(\Gamma) \otimes_{k} A(\Lambda)$.

To prove the first of these results we need a lemma on the graded centre.

Lemma 6.16. Let $R$ and $S$ be finitely generated graded connected $k$-algebras. Then the graded centre of $R \sqcup S$ is concentrated in degree 0 unless both $R$ and $S$ are isomorphic as ungraded rings to $k[x] / x^{2}$. 
Proof. If we fix bases $\left\{r_{i}: i \in J_{1}\right\}$ of $I(R)$ and $\left\{s_{i}: i \in J_{2}\right\}$ of $I(S)$ then $R \sqcup S$ has a basis consisting of the identity together with all words of the form $r_{i_{1}} s_{i_{2}} r_{i_{3}} \ldots r_{i_{n}}$, $s_{i_{1}} r_{i_{2}} s_{i_{3}} \ldots s_{i_{n}}, r_{i_{1}} s_{i_{2}} r_{i_{3}} \ldots s_{i_{n}}$ and $s_{i_{1}} r_{i_{2}} s_{i_{3}} \ldots r_{i_{n}}$. We refer to these words as being of types RR, SS, RS, and SR respectively.

Let $z$ be a homogeneous element of the graded centre of $R \sqcup S$. No word of type RR may appear in $z$, for given non-zero $s \in I(S)$ the product $s z$ would be non-zero and spanned by words beginning with an element of $s$ and therefore could not be equal to $\pm z s$. Similarly no words of type SS may appear in $z$.

If $r \in I(R)$ is non-zero then $r z$ is spanned by words of type RR and type RS, whereas $z r$ is spanned by words of type RR and type SR. Therefore only the type $\mathrm{RR}$ words may be non-zero. It follows that any type $\mathrm{RS}$ word in $z$ begins with an element of $A(R)$. By symmetry every word in $z$ of type RS begins with an element of $A(R)$ and ends with an element of $A(S)$, and every word of type $\mathrm{SR}$ in $z$ begins with an element of $A(S)$ and ends with an element of $A(R)$.

Again let $r \in I(R)$ be non-zero. $r z$ consists only of words beginning with $r$. The only elements of $I(R)$ beginning elements of $z r$ are in $A(R)$, and so every $r \in I(R)$ belongs to $A(R)$. It follows $I(R)^{2}=0$, and similarly $I(S)^{2}=0$. Comparing $r z$ and $z r$ for different choices of $r$ we see that $I(R)$ must be one-dimensional, and similarly $I(S)$.

If $R=k[x] / x^{2}$ and $S=k[y] / y^{2}$ then the graded centre of $R \sqcup S$ may be nontrivial, for example with $x$ and $y$ in degree 1 the element $x y+y x$ lies in the graded centre.

Proposition 6.14 can now be deduced: under its hypotheses the above lemma implies the graded centre is zero in positive degrees, but the image of $\phi_{k}$ lies in the graded centre of $E(\Lambda * \Gamma) \cong E(\Lambda) \sqcup E(\Gamma)$. This gives the first part of the proposition, and the last statement follows from [SS04, Proposition 4.4].

When $E(\Lambda) \cong E(\Gamma) \cong k[x] / x^{2}$, the map $\phi_{k}$ can be non-zero in positive degrees.

Example 6.17. Let $k$ have characteristic not two and $\Lambda=k[x]$ so that

$$
\begin{array}{ll}
E(\Lambda) \cong k[X] / X^{2} & E(\Lambda * \Lambda) \cong k\langle X, Y\rangle /\left(X^{2}, Y^{2}\right) \\
\mathrm{HH}^{0}(\Lambda) \cong k[x] & \operatorname{HH}^{1}(\Lambda) \cong k[x]
\end{array}
$$

and $\mathrm{HH}^{n}(\Lambda)$ is zero in higher degrees. Write $k\{S\}$ for the free graded commutative ring on a set of generators $S$ with specified degrees. Then the Hochschild cohomology ring of $\Lambda * \Lambda \cong k[x, y] / x y$ is

$$
\frac{k\left\{x_{0}, y_{0}, x_{1, r}, y_{1, r}, \xi_{2}, \xi_{3}: r \geq 1\right\}}{x_{0} y_{0}, x_{0} x_{1, r}=x_{1, r+1}, y_{0} x_{1, r}, y_{0} y_{1, r}=y_{1, r+1}, y_{0} x_{1, r}, x_{0} \xi_{i}, y_{0} \xi_{i}, \xi_{2} x_{1,1}=\xi_{3}=\xi_{2} y_{1,1}}
$$

where the first subscripts denote degrees. The map $\phi_{k}$ is the augmentation in degree zero, zero in odd degrees, and $\phi_{k}\left(\xi_{2}\right)=X Y+Y X$.

Remark 6.18. There does not seem to be a natural way to find $\mathrm{iHH}(\Lambda)$ as a subalgebra of $\mathrm{HH}(\Lambda * \Gamma)$. The inclusion $\iota: I(\Lambda) \hookrightarrow \Lambda * \Gamma$ is a map of $\Lambda * \Gamma$-bimodules, and the canonical projection $p_{\Lambda}$ induces $p_{\Lambda}^{e}:(\Lambda * \Gamma)^{e} \rightarrow \Lambda^{e}$, so functoriality of Ext gives a map

$$
\operatorname{Ext}_{p_{\Lambda}^{e}}^{n}\left(p_{\Lambda}, \iota\right): \operatorname{Ext}_{\Lambda^{e}}^{n}(\Lambda, I(\Lambda)) \rightarrow \operatorname{Ext}_{(\Lambda * \Gamma)^{e}}^{n}(\Lambda * \Gamma, \Lambda * \Gamma) .
$$

We could try to define a map $\mathrm{iHH}(\Lambda) \rightarrow \mathrm{HH}(\Lambda * \Gamma)$ by lifting an element of $\operatorname{ker} \phi_{k} \subset$ $\mathrm{iHH}(\Lambda)$ to an element of $\operatorname{Ext}_{\Lambda^{e}}(\Lambda, I(\Lambda))$ using (3), then mapping this to $\mathrm{HH}(\Lambda * \Gamma)$ with $\operatorname{Ext}_{p_{\Lambda}^{e}}^{n}\left(p_{\Lambda}, \iota\right)$. In order for this to be well defined we would require the latter map to kill elements of $\operatorname{Ext}_{\Lambda^{e}}(\Lambda, I(\Lambda))$ in the image of the connecting homomorphism $\omega_{\Lambda}$. This does not happen in general: it can be shown that

$$
\operatorname{Ext}_{p_{\Lambda}^{e}}\left(p_{\Lambda}, \iota\right) \circ \omega_{\Lambda}=\omega_{\Lambda * \Gamma} \circ \operatorname{Ext}_{p_{\Lambda}^{e}}\left(p_{\Lambda}, \mathrm{id}_{k}\right)
$$


where $\omega_{\Lambda * \Gamma}$ is the connecting homomorphism in the long exact sequence arising from applying $\operatorname{Hom}_{(\Lambda * \Gamma)^{e}}(\Lambda * \Gamma,-)$ to the exact sequence of $\Lambda * \Gamma$-bimodules

$$
0 \rightarrow \Lambda * \Gamma \rightarrow \Lambda \oplus \Gamma \rightarrow k \rightarrow 0
$$

where $\Lambda$ and $\Gamma$ are $\Lambda * \Gamma$-modules via $p_{\Lambda}$ and $p_{\Gamma}$, and the map $\Lambda * \Gamma \rightarrow \Lambda \oplus \Gamma$ is $1 \mapsto(1,1)$.

6.4. Proving Theorem 6.15. We begin by constructing the ideal $J$ referred to in the statement of Theorem 6.15. Let $\left((P \sqcup Q)_{*}, \delta_{*}\right)$ be a bimodule resolution for $\Lambda * \Gamma$ constructed as in Section 6.1. Write $\bar{P} * \bar{Q}$ for the subcomplex of $P \sqcup Q$ consisting of $(\Lambda * \Gamma)^{e}$ in degree zero and $\bar{P}_{n} \oplus \bar{Q}_{n}$ in degree $n>0$, with the induced differentials. In positive degrees this complex is equal to $\bar{P} \oplus \bar{Q}$. There is a short exact sequence

$$
0 \rightarrow \bar{P} * \bar{Q} \stackrel{j}{\rightarrow} P \sqcup Q \stackrel{\pi}{\rightarrow} E \rightarrow 0
$$

where $E$ is the quotient complex. Because $(\bar{P} * \bar{Q})_{n}$ is a summand of $(P \sqcup Q)_{n}$, the following sequence is also exact:

$0 \rightarrow \operatorname{Hom}_{(\Lambda * \Gamma)^{e}}(E, \Lambda * \Gamma) \rightarrow \operatorname{Hom}_{(\Lambda * \Gamma)^{e}}(P \sqcup Q, \Lambda * \Gamma) \rightarrow \operatorname{Hom}_{(\Lambda * \Gamma)^{e}}(\bar{P} * \bar{Q}, \Lambda * \Gamma) \rightarrow 0$.

Applying $\operatorname{Hom}_{(\Lambda * \Gamma)^{e}}(-, \Lambda * \Gamma)$ gives a long exact sequence

$$
\begin{aligned}
\cdots & \left.\stackrel{\omega}{\rightarrow} H^{n} \operatorname{Hom}_{(\Lambda * \Gamma)^{e}}(E, \Lambda * \Gamma)\right) \stackrel{\pi^{*}}{\rightarrow} \operatorname{HH}^{n}(\Lambda * \Gamma) \\
& \stackrel{j^{*}}{\rightarrow} H^{n}\left(\operatorname{Hom}_{(\Lambda * \Gamma)^{e}}(\bar{P} * \bar{Q}, \Lambda * \Gamma)\right) \stackrel{\omega}{\rightarrow} H^{n+1}\left(\operatorname{Hom}_{(\Lambda * \Gamma)^{e}}(E, \Lambda * \Gamma)\right) \stackrel{\pi^{*}}{\rightarrow} \cdots
\end{aligned}
$$

where $\omega$ is the connecting homomorphism. We now define $J$ to be im $\pi^{*}$. Elements of $\operatorname{im} \pi^{*}$ can be represented by cocycles on $P \sqcup Q$ that kill $\bar{P} * \bar{Q}$. As $\bar{P} * \bar{Q}$ is a subcomplex, such cocycles can be lifted to chain maps that kill $\bar{P} * \bar{Q}$. Hochschild cohomology is graded commutative so $J$ is an ideal of $\mathrm{HH}(\Lambda * \Gamma)$.

We now examine the connecting homomorphism $\omega$ more carefully. Let $a$ be an element of $H^{n}\left(\operatorname{Hom}_{(\Lambda * \Gamma)^{e}}(\bar{P} * \bar{Q}, \Lambda * \Gamma)\right)$ represented by a cocycle $\alpha: \bar{P}_{n} \rightarrow \Lambda * \Gamma$.

Identify $\Gamma^{e \uparrow}(\Lambda * \Gamma)^{e}$ with $(\Lambda * \Gamma)^{e}$ and $(\Lambda * \Gamma) \otimes_{k} k$ with $\Lambda * \Gamma$ so that $\left(1 \otimes \epsilon_{\Lambda * \Gamma}\right) \circ \bar{d}_{1}$ and $\left(\epsilon_{\Lambda * \Gamma} \otimes 1\right) \circ \bar{d}_{1}$ are maps $\bar{Q}_{1} \rightarrow \Lambda * \Gamma$. Then $\omega(a)$ is represented by the map $E_{n+1} \rightarrow \Lambda * \Gamma$ sending

$$
\begin{aligned}
& f \hat{\otimes} p \in \bar{Q}_{1} \hat{\otimes} \bar{P}_{n} \mapsto\left(1 \otimes \epsilon_{\Lambda * \Gamma}\right) \circ \bar{d}_{1}(f) \cdot \alpha(p) \\
& p \hat{\otimes} f \in \bar{P}_{n} \hat{\otimes} \bar{Q}_{1} \mapsto \alpha(p) \cdot\left(\epsilon_{\Lambda * \Gamma} \otimes 1\right) \circ \bar{d}_{1}(f)
\end{aligned}
$$

and killing all other summands (we have abused notation here: $f \hat{\otimes} p$ should be replaced by its image in $\left.E_{n+1}\right)$.

Lemma 6.19. A cocycle $\alpha: \bar{P}_{n} \rightarrow \Lambda * \Gamma$ represents an element of the kernel of the connecting homomorphism if and only if it has image contained in $I(\Lambda) \oplus A(\Gamma)$.

Proof. (15) shows that the element represented by such a cocycle has its image under the connecting homomorphism represented by the zero map. Conversely, suppose $\alpha: \bar{P}_{n} \rightarrow \Lambda * \Gamma$ is a cocycle and that the map (15) above is a coboundary. The image of this map is contained in $I(\Gamma)$, but in $E$ the differentials of the images of $f \hat{\otimes} p$ and $p \hat{\otimes} f \in E_{n}$ are in $E_{n-1} \cdot I(\Lambda)$ and $I(\Lambda) \cdot E_{n-1}$ respectively, by our hypothesis on the differential on $P_{*}$. It follows that any coboundary maps the images of $\bar{Q}_{1} \hat{\otimes} \bar{P}_{n-1}$ and $\bar{P}_{n-1} \hat{\otimes} \bar{Q}_{1}$ in $E$ into $I(\Lambda)$, hence (15) cannot be a coboundary unless it is zero.

The homology of $\left(\bar{P}_{*}, \bar{d}_{*}\right)$ computes

$$
\operatorname{Tor}_{*}^{\Lambda^{e}}\left((\Lambda * \Gamma)^{e}, \Lambda\right)=\operatorname{Tor}_{*}^{\Lambda^{e}}\left(\Lambda^{e} \oplus I(\Gamma) \otimes_{k} I(\Gamma), \Lambda\right)
$$


where the action of $\Lambda^{e}$ on $I(\Gamma) \otimes_{k} I(\Gamma)$ is trivial. Since $\Lambda^{e}$ is flat as a module over itself this is isomorphic to $I(\Gamma) \otimes_{k} I(\Gamma) \otimes_{k} \operatorname{Tor}_{*}^{\Lambda}(k, k)$ as a vector space, where we have used [CE56, X, Theorem 2.1] to identify $\operatorname{Tor}_{*}^{\Lambda^{e}}(k, \Lambda)$ with $\operatorname{Tor}_{*}^{\Lambda}(k, k)$. Furthermore

$$
\begin{aligned}
H^{n}\left(\operatorname{Hom}_{(\Lambda * \Gamma)^{e}}(\bar{P}, \Lambda * \Gamma)\right) & \cong H^{n}\left(\operatorname{Hom}_{\Lambda^{e}}(P, \Lambda \oplus I(\Gamma))\right) \\
& =\operatorname{Ext}_{\Lambda^{e}}^{n}(\Lambda, \Lambda) \oplus \operatorname{Ext}_{\Lambda^{e}}^{n}(\Lambda, I(\Gamma)) \\
& =\operatorname{HH}^{n}(\Lambda) \oplus \operatorname{Ext}_{\Lambda}^{n}(k, k) \otimes_{k} I(\Gamma) .
\end{aligned}
$$

The isomorphism is an application of the Eckmann-Shapiro lemma. The first summand on the last line comes from homomorphisms with image contained in $\Lambda \subset \Lambda * \Gamma$, and the second from those with image contained in $I(\Gamma) \subset \Lambda * \Gamma$.

We now have enough information for an additive decomposition of the Hochschild cohomology groups of $\mathrm{HH}(\Lambda * \Gamma)$.

Proposition 6.20. $\mathrm{HH}^{0}(\Lambda * \Gamma) \cong Z(\Lambda) * Z(\Gamma)$, and for $n>0$ there is an isomorphism of vector spaces

$\mathrm{HH}^{n}(\Lambda * \Gamma) \cong \mathrm{iHH}^{n}(\Lambda) \oplus \mathrm{iHH}^{n}(\Gamma) \oplus E^{n}(\Lambda) \otimes_{k} A(\Gamma) \oplus E^{n}(\Gamma) \otimes_{k} A(\Lambda) \oplus\left(\mathrm{im} \pi^{*}\right)_{n}$.

Proof. The statement about $\operatorname{HH}^{0}(\Lambda * \Gamma)$ is clear. In higher degrees, the long exact sequence (14) gives short exact sequences

$$
0 \rightarrow\left(\operatorname{im} \pi^{*}\right)_{n} \rightarrow \mathrm{HH}^{n}(\Lambda * \Gamma) \rightarrow(\operatorname{ker} \omega)_{n} \rightarrow 0 .
$$

Using Lemma 6.19 and (16) we can write $(\operatorname{ker} \omega)_{n}$ as a direct sum of $\mathrm{iHH}^{n}(\Lambda) \oplus$ $E^{n}(\Lambda) \otimes_{k} A(\Gamma)$ and the corresponding object for $\Gamma$. This completes the proof.

The isomorphism in this proposition is realised as follows: elements of $\mathrm{iHH}(\Lambda)$ correspond to cocycles mapping $\bar{P}_{*} \subset P \sqcup Q$ to $I(\Lambda) \subset \Lambda * \Gamma$ (and killing other summands). Elements of $E^{n}(\Lambda) \otimes_{k} A(\Gamma)$ correspond to cocycles mapping $\bar{P}_{*} \subset P \sqcup Q$ to $A(\Gamma) \subset \Lambda * \Gamma$ (and killing other summands). Elements of im $\pi^{*}$ are represented by cocycles on $P \sqcup Q$ that kill $\bar{P} * \bar{Q}$.

Returning to Example 6.17 where $\Lambda=k[x]$, we have $A(\Lambda)=0$ so only the iHH and $\mathrm{im} \pi^{*}$ terms appear in $\mathrm{HH}(\Lambda * \Lambda)$. The two copies of $\mathrm{iHH}(\Lambda)$ are generated by $x_{0}, x_{1, r}$ and $y_{0}, y_{1, r}$ for $r \geq 0$. The image of $\pi^{*}$ is the ideal $\left(\xi_{2}, \xi_{3}\right)$.

The last proposition means that there is a $k$-linear isomorphism between the groups in Theorem 6.15, so we only need to show that it can be made multiplicative. A careful reading of the proof of [SS04, Proposition 4.4] shows that it implies the following result.

Proposition 6.21. Let $\Delta$ be an augmented algebra and $\left(P_{*}, d\right)$ be a bimodule resolution of $\Delta$ with the property that im $d \subset I(\Delta) \cdot P+P \cdot I(\Delta)$. Let $J \subset I(\Delta)$ be an ideal of $\Delta$. If $\xi, \eta \in \mathrm{HH}(\Delta)$ can be represented by cocycles $P_{*} \rightarrow \Delta$ with images contained in $I(\Delta)$ and $J$ respectively, then $\xi \eta$ can be represented by a cocycle with image contained in $I(\Delta) \cdot J+J \cdot I(\Delta)$.

It follows immediately that elements of $\mathrm{HH}(\Lambda * \Gamma)$ which are identified with elements of $E(\Lambda) \otimes_{k} A(\Gamma)$ under the isomorphism in Proposition 6.20 have product zero with any element of $\operatorname{ker} \phi_{k}: \mathrm{HH}(\Lambda * \Gamma) \rightarrow \Lambda * \Gamma$. In particular, this verifies the last sentence of Theorem 6.15. The only thing left to prove is that the elements of $\mathrm{HH}(\Lambda * \Gamma)$ identified with elements of $\mathrm{iHH}(\Lambda)$ and $\mathrm{iHH}(\Gamma)$ in Proposition 6.20 multiply, modulo $R$, like $\mathrm{iHH}(\Lambda) * \mathrm{iHH}(\Gamma)$. That elements of the copies of $I(\mathrm{iHH}(\Lambda))$ and $I(\mathrm{iHH}(\Gamma))$ have product zero is clear. Given an element of $\mathrm{iHH}(\Lambda)$ we now show how to associate an element of $\mathrm{HH}(\Lambda * \Gamma)$ in a manner that induces a multiplicative map $\mathrm{iHH}(\Lambda) \rightarrow \mathrm{HH}(\Lambda * \Gamma) / R$ whose image modulo $R$ is the copy of $\mathrm{iHH}(\Lambda)$ in $\mathrm{HH}(\Lambda * \Gamma)$ identified in Proposition 6.20.

Let $f: P_{n} \rightarrow \Lambda$ be a cocycle representing an element of $\operatorname{iHH}(\Lambda)$, so that the image of $f$ is contained in $I(\Lambda)$. Lift $f$ to a chain map $f_{*}$ on $P_{*}$. We now build a 
chain map $F_{*}: P \sqcup Q \rightarrow P \sqcup Q$ using $\bar{f}_{*}$ that lifts the cocycle $\hat{f}:(P \sqcup Q)_{n} \rightarrow \Lambda * \Gamma$ sending $x \otimes_{\Lambda} p \otimes_{\Lambda} y \in \bar{P}_{n}$ to $x f(p) y \in \Lambda * \Gamma$, and killing all other summands. The map $F_{*}$ is defined as follows:

$$
\begin{aligned}
& F_{*}(p)=\bar{f}_{m}(p) \\
& F_{*}(p \hat{\otimes} \cdots \hat{\otimes} q)=\bar{f}_{m}(p) \hat{\otimes} \cdots \hat{\otimes} q \quad \text { for } p \hat{\otimes} \cdots \hat{\otimes} q \in \bar{P}_{m} \hat{\otimes} \cdots \hat{\otimes} \bar{Q}_{r} \text { and } m>n \text {. } \\
& F_{*}(q \hat{\otimes} \cdots \hat{\otimes} p)=q \hat{\otimes} \cdots \hat{\otimes} \bar{f}_{m}(p) \quad \text { for } q \hat{\otimes} \cdots \hat{\otimes} p \in \bar{Q}_{r} \hat{\otimes} \cdots \hat{\otimes} \bar{P}_{m} \text { and } m>n \text {. } \\
& F_{*}\left(p_{1} \hat{\otimes} \cdots \hat{\otimes} p_{2}\right)=\bar{f}_{m}\left(p_{1}\right) \hat{\otimes} \cdots \hat{\otimes} p_{2}+p_{1} \hat{\otimes} \cdots \hat{\otimes} \bar{f}_{m}\left(p_{2}\right) \\
& \text { for } p_{1} \hat{\otimes} \cdots \hat{\otimes} p_{2} \in \bar{P}_{m_{1}} \hat{\otimes} \cdots \hat{\otimes} \bar{P}_{m_{2}} \text { and } m_{1}, m_{2}>n \text {. } \\
& F_{*}\left(p \hat{\otimes} q_{1} \hat{\otimes} \cdots \hat{\otimes} q_{2}\right)=\left(\left(1 \otimes \epsilon_{\Lambda * \Gamma}\right) \circ \bar{f}_{0}\right)(p) \cdot q_{1} \hat{\otimes} \cdots \hat{\otimes} q_{2} \\
& \text { for } p \hat{\otimes} q_{1} \hat{\otimes} \cdots \hat{\otimes} q_{2} \in \bar{P}_{n} \hat{\otimes} \bar{Q}_{r} \hat{\otimes} \cdots \hat{\otimes} \bar{Q}_{s} . \\
& F_{*}\left(q_{1} \hat{\otimes} \cdots \hat{\otimes} q_{2} \hat{\otimes} p\right)=\left(q_{1} \hat{\otimes} \cdots \hat{\otimes} q_{2} \cdot\left(\left(\epsilon_{\Lambda * \Gamma} \otimes 1\right) \circ \bar{f}_{0}\right)(p)\right. \\
& \text { for } q_{1} \hat{\otimes} \cdots \hat{\otimes} q_{2} \hat{\otimes} p \in \bar{Q}_{r} \hat{\otimes} \cdots \hat{\otimes} \bar{Q}_{s} \hat{\otimes} \bar{P}_{n} \text {. } \\
& F_{*}\left(p_{1} \hat{\otimes} q_{1} \hat{\otimes} \cdots \hat{\otimes} q_{2} \hat{\otimes} p_{2}\right)= \\
& \left(\left(1 \otimes \epsilon_{\Lambda * \Gamma}\right) \circ \bar{f}_{0}\right)(p) \cdot q_{1} \hat{\otimes} \cdots \hat{\otimes} q_{2} \hat{\otimes} p_{2}+p_{1} \hat{\otimes} q_{1} \hat{\otimes} \cdots \hat{\otimes} q_{2} \cdot\left(\left(\epsilon_{\Lambda * \Gamma} \otimes 1\right) \circ \bar{f}_{0}\right)(p) \\
& \text { for } p_{1} \hat{\otimes} q_{1} \hat{\otimes} \cdots \hat{\otimes} q_{2} \hat{\otimes} p_{2} \in \bar{P}_{n} \hat{\otimes} \bar{Q}_{r} \hat{\otimes} \cdots \hat{\otimes} \bar{Q}_{s} \hat{\otimes} \bar{P}_{n} \text {. }
\end{aligned}
$$

As before, we regard $\left(1 \otimes \epsilon_{\Lambda * \Gamma}\right) \circ \bar{f}_{0}$ and $\left(\epsilon_{\Lambda * \Gamma} \otimes 1\right) \circ \bar{f}_{0}$ as maps $\bar{P}_{m} \rightarrow \Lambda * \Gamma$. Finally, $F_{*}$ is zero on all other summands of $P \sqcup Q$. The reader may verify that $F_{*}$ is a chain map lifting $\hat{f}$. Write $c\left(f_{*}\right)$ for the chain map $F_{*}$ built from $f_{*}$.

Lemma 6.22. $c$ induces a well-defined multiplicative map $\mathrm{iHH}(\Lambda) \rightarrow \mathrm{HH}(\Lambda * \Gamma) / R$.

Proof. For well-definedness, we must show that if $f$ is a coboundary then $c\left(f_{*}\right)$ is zero in $\mathrm{HH}(\Lambda * \Gamma) / R$. Suppose $f=g \circ d_{n}: P_{n} \rightarrow \Lambda$ is a coboundary. Then $\hat{g}$ and $\hat{f} \circ \delta$ agree on $\bar{P} * \bar{Q}$, so their difference is a cocycle that is zero on $\bar{P} * \bar{Q}$ and which therefore represents an element of $R$. The map is multiplicative because if $f_{*}$ and $g_{*}$ are two chain maps on $P_{*}$ then $c\left(f_{*} \circ g_{*}\right)-c\left(f_{*}\right) \circ c\left(g_{*}\right)$ is zero on $\bar{P} * \bar{Q}$.

This completes the proof of Theorem 6.15 .

\section{ACKNOWLEDGEMENTS}

I would like to thank the referee for numerous suggestions on how to improve the presentation of the paper, and for the remark on Koszul duality after Proposition 6.14 .

\section{REFERENCES}

[Ben98] D. Benson, Representations and cohomology II: Cohomology of groups and modules, 2nd ed., Cambridge studies in advanced mathematics, vol. 31, Cambridge University Press, 1998.

[CE56] H. Cartan and S. Eilenberg, Homological algebra, Princeton University Press, 1956.

[HS71] P. Hilton and U. Stammbach, A course in homological algebra, Springer-Verlag, New York, 1971, Graduate Texts in Mathematics, Vol. 4.

[Lod98] J-L. Loday, Cyclic homology, 2nd ed., Grundlehren der Mathematischen Wissenschaften, vol. 301, Springer-Verlag, Berlin, 1998.

[Mac71] S. MacLane, Categories for the working mathematician, Springer-Verlag, New York, 1971, Graduate Texts in Mathematics, Vol. 5.

[Moo09] W. Moore, Cohomology over fiber products of local rings, J. Algebra 321 (2009), no. 3, $758-773$.

[PP05] A. Polishchuk and L. Positselski, Quadratic algebras, University Lecture Series, vol. 37, American Mathematical Society, Providence, RI, 2005.

[SS04] N. Snashall and Ø. Solberg, Support varieties and Hochschild cohomology rings, Proc. London Math. Soc. (3) 88 (2004), no. 3, 705-732. 
[Tow09] M. Towers, Endomorphism algebras of transitive permutation modules for p-groups, Archiv der Math. 92 (2009), no. 3, 215-227.

Mathematical Institute, University of Oxford, 24-29 St. Giles, Oxford OX1 3LB, United Kingdom

E-mail address: towers@maths.ox.ac.uk 
[Tow09] M. Towers, Endomorphism algebras of transitive permutation modules for p-groups, Archiv der Math. 92 (2009), no. 3, 215-227.

Mathematical Institute, University of Oxford, 24-29 St. Giles, Oxford OX1 3LB, United Kingdom

E-mail address: towers@maths.ox.ac.uk 